\title{
Design and Performance of the NASA SCEPTOR Distributed Electric Propulsion Flight Demonstrator
}

\author{
Nicholas K. Borer ${ }^{1}$, Michael D. Patterson ${ }^{2}$, Jeffrey K. Viken ${ }^{3}$, Mark D. Moore ${ }^{4}$ \\ NASA Langley Research Center, Hampton, Virginia 23681 \\ Sean Clarke ${ }^{5}$, Matthew E. Redifer ${ }^{6}$ \\ NASA Armstrong Flight Research Center, Edwards, California, 93523 \\ Robert J. Christie ${ }^{7}$ \\ NASA Glenn Research Center, Cleveland, Ohio, 44135 \\ Alex M. Stoll ${ }^{8}$, Arthur Dubois ${ }^{9}$, JoeBen Bevirt ${ }^{10}$ \\ Joby Aviation, Santa Cruz, California, 95060
}

Andrew R. Gibson ${ }^{11}$, Trevor J. Foster ${ }^{12}$, Philip G. Osterkamp ${ }^{13}$

Empirical Systems Aerospace, Inc., Pismo Beach, California 93448

\begin{abstract}
Distributed Electric Propulsion (DEP) technology uses multiple propulsors driven by electric motors distributed about the airframe to yield beneficial aerodynamic-propulsion interaction. The NASA SCEPTOR flight demonstration project will retrofit an existing internal combustion engine-powered light aircraft with two types of DEP: small "high-lift" propellers distributed along the leading edge of the wing which accelerate the flow over the wing at low speeds, and larger cruise propellers co-located with each wingtip for primary propulsive power. The updated high-lift system enables a $2.5 x$ reduction in wing area as compared to the original aircraft, reducing drag at cruise and shifting the velocity for maximum lift-to-drag ratio to a higher speed, while maintaining low-speed performance. The wingtip-mounted cruise propellers interact with the wingtip vortex, enabling a further efficiency increase that can reduce propulsive power by $10 \%$. A tradespace exploration approach is developed that enables rapid identification of salient trades, and subsequent creation of SCEPTOR demonstrator geometries. These candidates were scrutinized by subject matter experts to identify design preferences that were not modeled during configuration exploration. This exploration and design approach is used to create an aircraft that consumes an estimated $4.8 x$ less energy at the selected cruise point when compared to the original aircraft.
\end{abstract}

${ }^{1}$ Technical Lead/Aerospace Engineer, Aeronautics Systems Analysis Branch, MS 442, AIAA Senior Member.

${ }^{2}$ Aerospace Engineer, Aeronautics Systems Analysis Branch, MS 442, AIAA Member.

${ }^{3}$ Aerospace Engineer, Aeronautics Systems Analysis Branch, MS 442, AIAA Member.

${ }^{4}$ Principal Investigator, Aeronautics Systems Analysis Branch, MS 442, AIAA Member.

${ }^{5}$ Co-Principal Investigator, Advanced Systems Development Branch, P.O. Box 273, MS 4840D.

${ }^{6}$ Electrical Engineer, Advanced Systems Development Branch, P.O. Box 273, MS 4840D, AIAA Member.

${ }^{7}$ Aerospace Engineer, Thermal Systems Branch, 21000 Brookpark Rd, MS 86/12.

${ }^{8}$ Aeronautical Engineer, 340 Woodpecker Ridge, AIAA Member.

${ }^{9}$ Aeronautical Engineer, 340 Woodpecker Ridge, AIAA Member.

${ }^{10}$ Founder, 340 Woodpecker Ridge.

${ }^{11}$ President/Aerospace Engineer, P.O. Box 595, AIAA Senior Member.

${ }^{12}$ Aerospace Engineer, P.O. Box 595, AIAA Member.

${ }^{13}$ Aerospace Engineer, P.O. Box 595, AIAA Member. 


\section{Nomenclature}

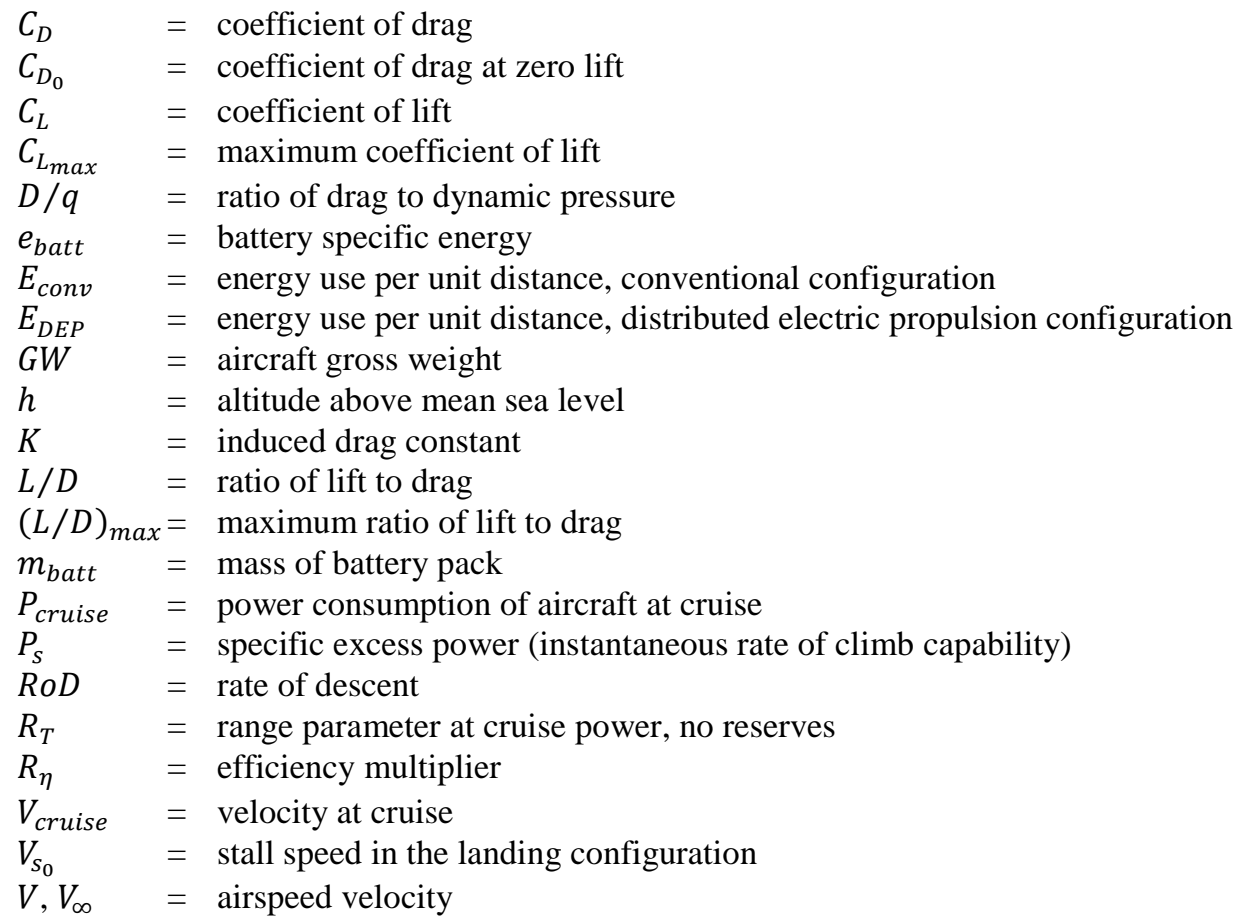

\section{Introduction}

The use of electric motors for aircraft propulsion is a topic of increasing interest in the aviation community, largely due to the significant increase in efficiency of these motors vs. traditional internal combustion engines. For light aircraft, these motors are more compact, lighter, quieter, and more reliable than the reciprocating combustion engines that are currently used for primary propulsion [1]. Ongoing development, research, and eventual production projects are focusing on the low-power, low-range, limited utility platforms dedicated to the flight training market, as seen in the Airbus E-Fan [2] and Pipistrel Alpha Electro [3]. These are seen as stepping-stone platforms by their parent companies for entrance into larger, more powerful aircraft.

One of the most significant barriers to adoption of electric propulsion in aircraft is the weight of the onboard energy storage. Current battery technologies yield 60-100x less energy stored per unit mass as compared to typical aircraft fuels [4]. Even with a threefold increase in efficiency, this is a prohibitive mass penalty for a simple powerplant retrofit to yield the same payload and range performance as the gasoline-powered counterpart. However, this impact is lessened if the mission capabilities of the aircraft are matched to actual use. For example, McDonald shows two use cases where current missions are performed by aircraft that have too much payload-range capability [5]; it is these "short-haul" missions that will likely be the early beneficiaries of electric propulsion.

While electric aircraft may be designed to a different requirements set, they will also benefit from changes in propulsion integration. The relatively scale-invariant nature of efficiency vs. power level (and motor size) tends to enable more distributed propulsion architectures than seen with typical thermodynamic cycles used for propulsion [6, 7]. In particular, Distributed Electric Propulsion (DEP) architectures can yield a net benefit in total efficiency due to synergistic airframe-propulsive coupling [8]. This further reduces the penalty associated with a heavier onboard energy storage mechanism, as the overall aerodynamic efficiency can be improved.

NASA has been investigating the benefits of DEP technology, including development of ground test articles, and more recently on the design of a flight demonstrator to establish and verify some of the potential benefits associated with this technology [9]. This paper will describe the tradespace exploration, conceptual design, and performance estimates of the proposed flight demonstrator for the NASA Scalable Convergent Electric Propulsion Technology Operations Research (SCEPTOR) project. 


\section{A. Distributed Electric Propulsion}

Distributed Electric Propulsion is not a single integration strategy, but rather a suite of possible airframepropulsion integrations that can yield net efficiency benefits using different, targeted approaches that may not otherwise be achievable with a few, large propulsion units. Distributed Propulsion may appear to be a better moniker; however, electric propulsion is an enabler of Distributed Propulsion due to the relatively high power-toweight ratio of the electric motors and their relatively scale-invariant nature. Hence, Distributed Propulsion is referred to hereon as Distributed Electric Propulsion.

A concept that uses DEP is shown in Figure 1. Here, three different types of DEP are encountered. The propellers along the leading edge of the wing are used to enhance high-lift performance by increasing the dynamic pressure over the wing at low speeds, enabling a smaller wing. The propellers at the wingtips are used for primary propulsion, and rotate opposite of the wingtip vortex, increasing propulsive efficiency. The single small propeller at the aft end of the fuselage is used to accelerate the slow moving air along the fuselage boundary layer as a means to "cancel" out fuselage drag. SCEPTOR is primarily interested in the first two incarnations: dynamic pressure scaling to enhance low-speed lift, and wingtip-mounted primary propulsors for increased efficiency during cruise.

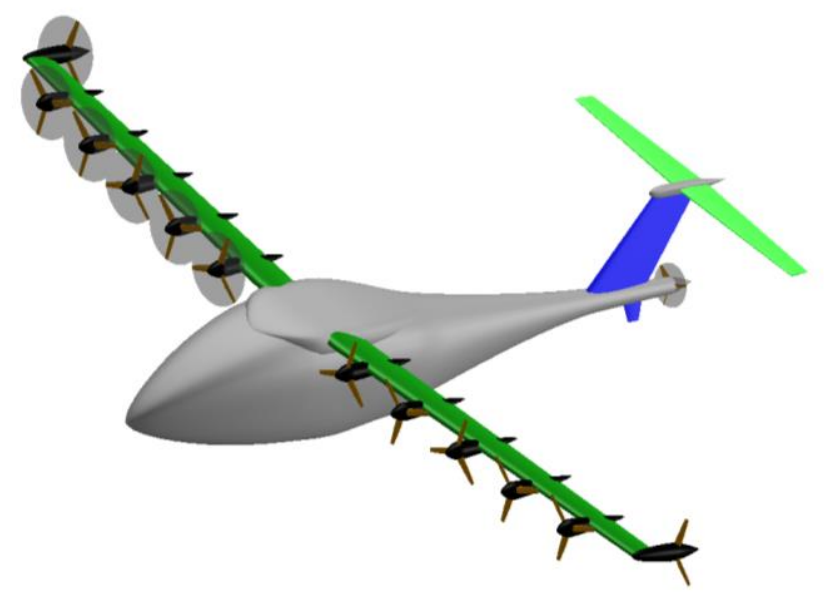

Figure 1: Notional Concept with Three Different Implementations of Distributed Electric Propulsion Technology

One of the major integration benefits of DEP is the use of propellers distributed along the wingspan to enhance the dynamic pressure over the wing at slow speeds. This enables the use of smaller wings for greater high-speed cruise efficiency without compromising low-speed performance, as is necessary for takeoff and landing (particularly out of confined areas). This is a major concern for light aircraft, which are often designed with oversized wings as compared to their target cruise speeds. This is because the oversized wing has a much lower velocity where the airframe lift-to-drag ratio is maximized than where the aircraft designer wishes to cruise.

The potential benefit is shown in Figure 2. Here, a notional parabolic drag polar is assumed - all data points use identical zero-lift drag coefficient $C_{D_{0}}$ and induced drag constant $K$ terms, such that total drag coefficient $C_{D}=$ $C_{D_{0}}+K C_{L}{ }^{2}$, where $C_{L}$ is the aircraft lift coefficient. Three different wing loadings are derived, according to three different maximum lift coefficients $C_{L_{\max }}$, to meet the same stall speed requirement in the landing configuration $V_{S_{0}}$ of 61 knots calibrated airspeed (KCAS). The maximum lift coefficients varied as $1 \mathrm{x}, 2 \mathrm{x}$, and $3 \mathrm{x}$ multiples of a notional light aircraft $C_{L_{\max }}$ of 1.8, which is representative for this class of aircraft. The plot shows the lift-to-drag ratio $L / D$ versus airspeed velocity of the three otherwise identical aircraft with progressively smaller wing area. Given identical drag polars, the maximum lift-to-drag ratio, $(L / D)_{\max }$, occurs at a higher velocity as the maximum lift capability of the wing increases for a given stall speed. This illustrates one of the great benefits of using DEP to augment the lift over the wing at lower speeds - the speed at which the most efficient cruise occurs (the speed where $L / D$ is maximized) is higher. The high-lift propellers are designed for low-speed operation to enhance dynamic pressure over the wing, rather than for propulsive efficiency at cruise. They are powered down and folded back against their nacelles for low drag at the high-speed cruise point, which is when the flowfield around the wing does not need additional augmentation from the high-lift propellers. Hence, the primary purpose of these propellers is simply to augment the lift of the wing at low speed - they are not designed to provide primary propulsion. 


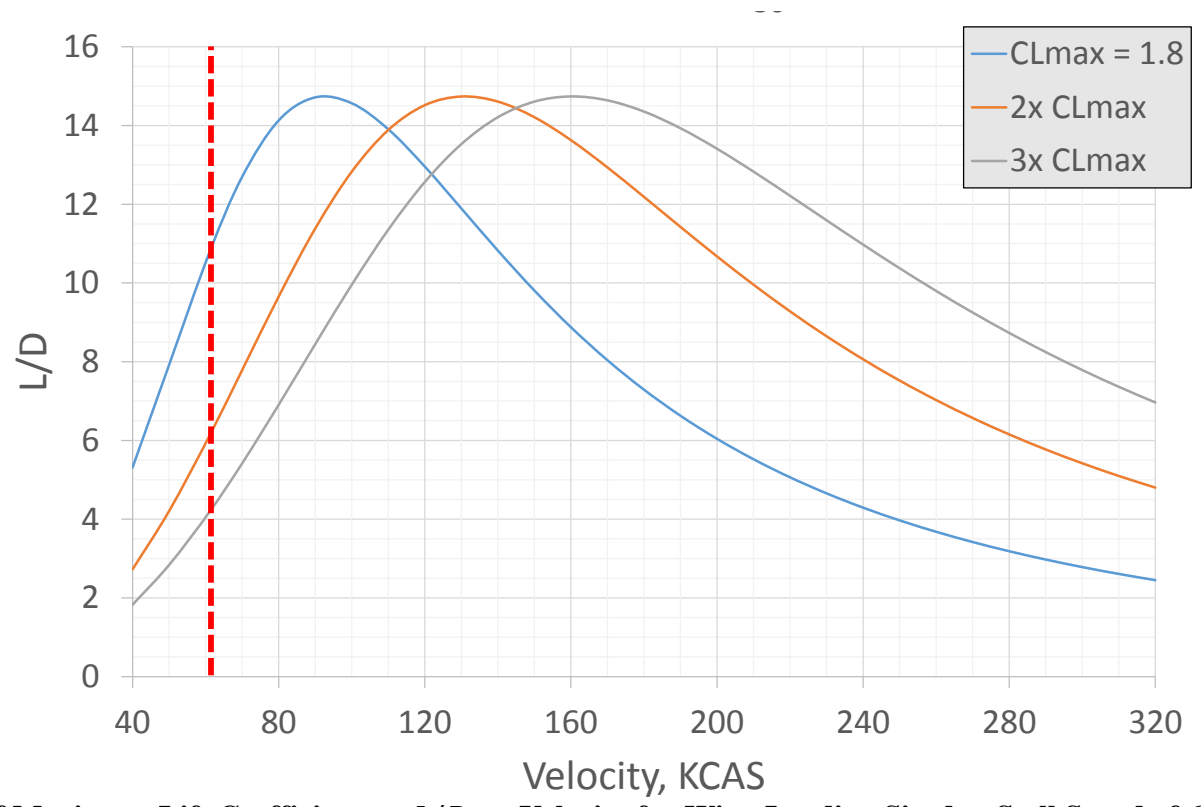

Figure 2: Effect of Maximum Lift Coefficient on $L / D$ vs. Velocity for Wing Loading Sized to Stall Speed of 61 KCAS

The wingtip-mounted propellers provide primary propulsion. Mounting at the wingtips enables these propellers to be spun against the tip vortex, which results in an increase in propulsive efficiency or a reduction in induced drag, depending on the placement of the props (tractor vs. pusher) and the method of bookkeeping [10]. Total drag reductions of over $10 \%$ can be realized, particularly for DEP configurations. The wing of aircraft using high-lift propellers for low-speed lift augmentation will be more heavily loaded at cruise, so the wingtip vortex will be stronger than aircraft with more traditional lightly loaded wings.

\section{Project Goals and Constraints}

SCEPTOR will retrofit an existing aircraft design with DEP technologies, rather than develop a clean-sheet DEP configuration. This enables a significant reduction in demonstration cost and schedule, and also helps to mitigate some risks by associating the systems not modified by DEP with proven designs. The retrofit approach allows for an objective comparison of the aircraft prior to retrofit with the DEP configuration of the same aircraft.

Several incremental demonstrations, dubbed "Mods" (for "modification") are utilized on SCEPTOR to mitigate risk throughout the project, as shown in Figure 3. The use of an existing vehicle for retrofit, along with the incremental demonstrations, help shape the overall vehicle requirements and "desirements" that are critical to sizing the DEP wing and propulsion systems.

\section{A. Driving Considerations}

SCEPTOR's primary objective is to demonstrate a large reduction in energy consumption compared to a conventionally-powered aircraft of the same size class in cruise. The "stretch" goal is to demonstrate a fivefold reduction in energy consumption, and the minimum threshold goal is demonstration of a $3.5 \mathrm{x}$ reduction. The lower limit is based on the estimated change in efficiency from the powerplant itself. A typical aviation internal combustion powerplant has an efficiency of approximately $30 \%$ when referenced to the lower heating value of the fuel stored onboard the aircraft, whereas modern non-cryogenic electric motors can exceed $90 \%$ in combined motorcontroller-battery discharge efficiency. This results in an approximately threefold reduction in energy consumption for the same size and speed class of vehicle. Thus, the lower limit of 3.5x reduction would reflect the full benefit of switching propulsion systems as well as a small synergistic benefit from DEP - otherwise, DEP would simply not "buy" its way onto the airframe. Preliminary investigations show that the propulsive-airframe coupling benefits of DEP can exceed a $1.5 x$ multiplier, largely by enabling higher wing loading through the use of high-lift propellers, and the integration of wingtip-mounted cruise propellers, as described above. Hence, the project adopted the goal of a total of a fivefold decrease in cruise energy consumption, referenced to the energy stored onboard the aircraft (e.g., not accounting for distribution chains to the aircraft - so called "well-to-wake" energy consumption). 


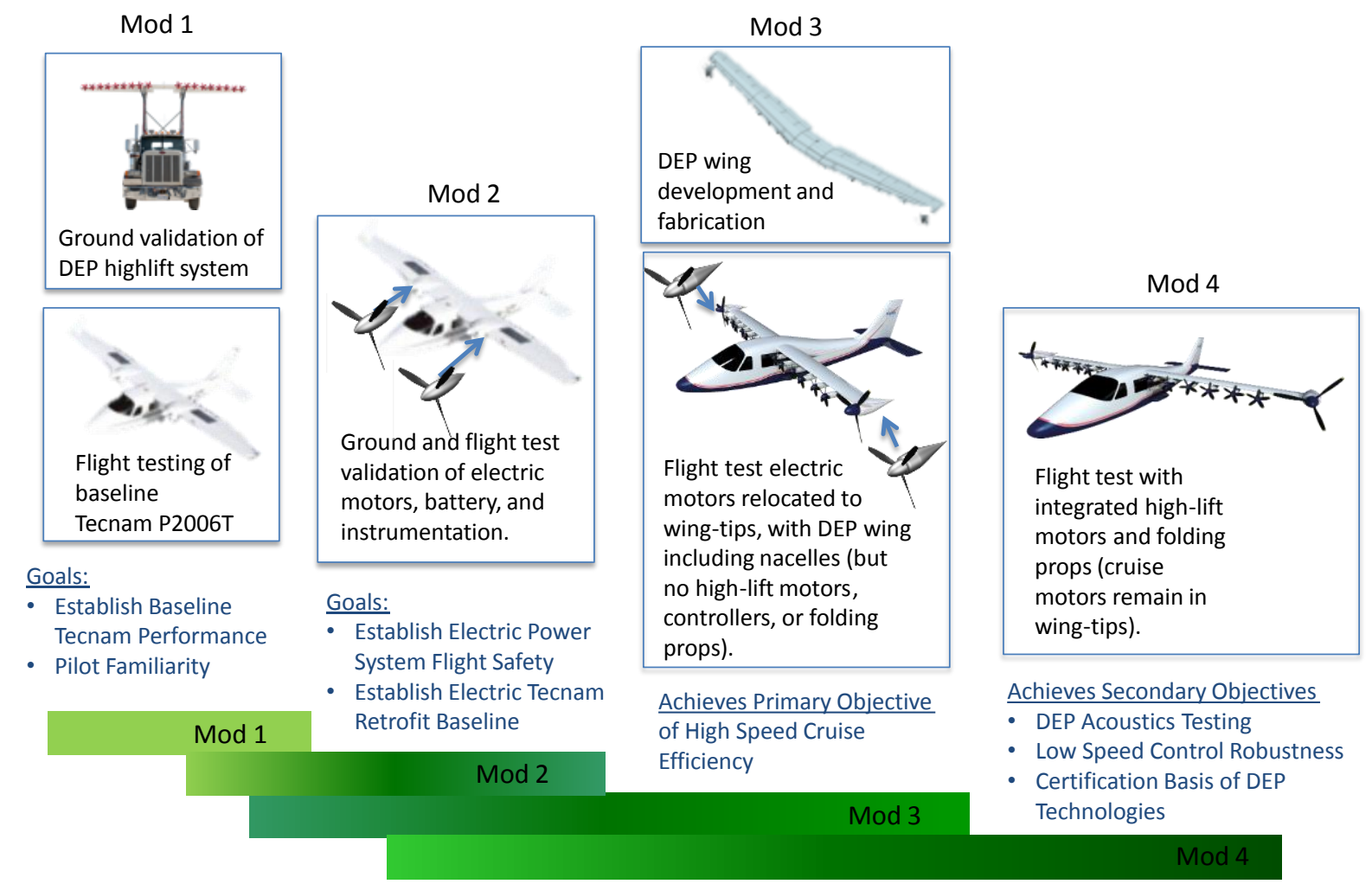

Figure 3: SCEPTOR Incremental Demonstrations ("Mods")

\section{Range-Specific Energy Consumption}

The primary objective for reduction in cruise energy needs to be anchored in a measurable, repeatable fashion. For a given fuselage size and stall speed, a DEP wing will have higher cruise efficiency at higher velocities than a traditional wing (as shown from Figure 1 earlier), hence, it makes little sense to compare the cruise efficiency at a single altitude/velocity point. Rather, the comparison can be made across the flight envelopes of the two vehicles over the entire range of the intersection of altitude/velocity combinations achievable by both aircraft. The primary metric, the range efficiency multiplier $R_{\eta}$, is determined from

$$
R_{\eta}=E_{D E P} / E_{c o n v}
$$

where $E_{D E P}$ is the range-specific energy consumption of the DEP configuration and $E_{c o n v}$ is the range-specific energy consumption of the baseline conventional configuration. The range-specific energy consumption is determined by

$$
E_{(D E P \text { or conv })}=P_{\text {cruise }} / V_{\text {cruise }}
$$

where $P_{\text {cruise }}$ is the rate of energy consumed from the onboard storage system at cruise, and $V_{\text {cruise }}$ is the airspeed velocity. The resulting quantity is expressed in energy used per unit distance. For comparison with traditional battery and flight vehicle nomenclature, where energy storage is frequently expressed in $W \cdot h r$ and distance in $\mathrm{nm}$, the cruise energy consumption rate is expressed in $W \cdot h r / n m$. The value of $P_{\text {cruise }}$ is found in the conventional aircraft by multiplying the fuel flow rate by the lower heating value of the fuel consumed, whereas it is found in the DEP aircraft by multiplying the voltage and current at the battery in the cruise condition.

\section{Altitude}

Cruise demonstration test points will be limited to the capability of the airframe and energy system. Batteries, while they are enjoying sizable investment and continual improvement in capacity, remain significantly less energydense than traditional hydrocarbon fuels in the near-term. Despite the advantages in cruise energy consumption, it is unlikely that the retrofit demonstration aircraft (which must use near-term battery technology) will have much 
endurance without a substantial mass penalty from the battery system. Hence, cruise altitude test points will need to be low enough that the aircraft will have sufficient energy to climb to altitude, fly the test point, and return without exhausting the energy supply (and with sufficient reserves). This is likely not a driving constraint, since the retrofit aircraft will likely be unpressurized, and there will be additional safety hazards and crew limitations by requiring oxygen or a pressure suit for the test pilot.

\section{Payload}

The aircraft will be piloted, as this tends to be a more cost-effective way to conduct a flight demonstration for a vehicle of moderate size. Thus, the retrofit aircraft will need to have sufficient payload capacity for a pilot, the pilot's necessary operational and emergency equipment, and any additional test instrumentation needed to capture system performance data. The initial sizing of this configuration assumed that pilot, equipment, and instrumentation weight equaled 400 pounds.

\section{Wing Size}

Aircraft wing sizing is a compromise between low-speed and high-speed performance. As noted earlier, DEP allows for higher wing loading for a given stall speed than traditional configurations, much like any other high-lift device. To maintain objectivity in the comparison between the conventional and DEP configurations, the DEP wing is sized to the same stall speed as the conventional configuration. However, given that the DEP configuration may have a small change in gross weight compared to the conventional configuration, the stall speed requirement is normalized to change in gross weight such that

$$
V_{S_{0}, D E P}=V_{S_{0}, \text { conv }} \sqrt{G W_{D E P} / G W_{\text {conv }}}
$$

where $V_{s_{0}}$ is the stall speed in the landing configuration, $G W$ is gross weight, and the subscripts $D E P$ and conv are used to denote the DEP and conventional configurations, respectively.

One consequence of changing the shape of the $L / D$ vs. velocity curve per Figure 1 is the change in power-off rate of descent for the aircraft, which is determined from

$$
R o D=\frac{V_{\infty}}{L / D}
$$

where $R o D$ is the power-off rate of descent and $V_{\infty}$ is the current airspeed velocity. Aircraft that otherwise have the same $(L / D)_{\max }$ but higher loaded wings will have a higher minimum power-off rate of descent. This can result in higher loads for the landing gear during hard landings. For this reason, 14 CFR Part 23.473 [11] specifies sink rate velocities for landing gear load requirements as a function of wing loading. Thus, the minimum sink rate for a DEP wing will very likely be higher than the unmodified aircraft. Luckily, certified aircraft are designed to exceed required landing gear load margins as a hedge against future gross weight increases or poor pilot technique. The retrofit aircraft landing gear load margin over required certification values may ultimately determine how much DEP can "shrink" the wing (alternatively, SCEPTOR could design stronger landing gear, though this may be cost- and schedule-prohibitive). This is not necessarily a true design constraint, as the aircraft will be piloted by highly qualified NASA test pilots, minimizing the probability of a hard landing. However, SCEPTOR needs to track how much landing gear load margin is consumed with respect to certified aircraft, as this represents the magnitude of the landing loads risk that needs to be mitigated operationally vs. by design.

\section{Failures}

One of the benefits of DEP configurations is the potential for massive redundancy in the event of a single motor failure. Though the high-lift propellers are not primarily designed for propulsion, they do provide some thrust as a by-product of their lift-enhancing approach. However, SCEPTOR's incremental approach to flight test involves configurations that will not be able to take advantage of this feature of DEP. In particular, SCEPTOR will flight test a version with the cruise propellers only (located at the vehicle wingtips). This would result in motor-out moments that cannot be trimmed at low speeds with traditional control surface deflections without significant increases in vertical tail area. This results in a minimum control speed that is well over the takeoff and approach speeds.

As a mitigation, SCEPTOR is conducting these flight tests at Dryden Field, co-located with NASA Armstrong Flight Research Center (AFRC) and Edwards Air Force Base, which is centered in a large, flat, dry lakebed with few obstructions for miles in any direction. In the unlikely event of a total wingtip motor failure, the procedure will be similar to that of a single-engine aircraft: glide to a safe landing on the lakebed (or return to the airfield if sufficient 
altitude exists), in this case with the opposite motor at low power. As such, no engine-out climb requirements are in place for SCEPTOR. It should be noted that this is simply an artifact of the development process - future practical DEP aircraft will all be designed such that the high-lift propellers can be configured as part of the control architecture for the appropriate combinations of motor-out scenarios.

With this mitigation, SCEPTOR is not unlike the design paradigm associated with single-engine aircraft. The Federal Aviation Administration performance requirements for climb for single-engine aircraft are outlined in 14 CFR 23.65, which states that a climb gradient of $8.3 \%$ is required for landplanes and $6.7 \%$ for seaplanes [11]. Simply put, the climb gradient for single-engine aircraft ensures enough climb performance is available to minimize exposure time to engine failure close to the ground, when there may not be enough altitude to execute a 180-degree turn to return to the field. Seaplanes are allowed a lower climb gradient because they tend to takeoff from more open environments (lakes and rivers), giving more opportunity to glide down back to a safe surface even when an engine failure occurs soon after takeoff. Given the dearth of obstacles surrounding SCEPTOR's planned test site, the seaplane climb gradient serves as a de facto requirement for takeoff climb performance.

DEP configurations favor higher cruise speeds than conventional aircraft due to the highly loaded wing; hence, retractable gear configurations are preferable to mitigate parasite drag at higher speeds. From a failure perspective, low-wing configurations may not be desirable - in the event of a gear-up landing, significant damage could occur to numerous high-lift propellers and motors. Though survivable, this could result in a significant budget and schedule setback for the demonstrator project. Mid- or high-wing configurations would be less likely to damage the high-lift or tip propellers.

\section{Crosswind}

Mid- and high-wing configurations are also desirable for the wingtip cruise propellers. Higher wing locations generally provide more propeller clearance between the ground and propeller tips when the vehicle is taxiing or otherwise in contact with the ground. Crosswind landing procedures may need to be operationally restricted depending on the wing span and tip propeller diameter, as a typical operational technique for landing in a crosswind includes landing with a slight bank into the wind. Overall, allowable bank angle at touchdown serves as a constraint on the combination of wing span and propeller diameter.

\section{B. Baseline Airframe Selection}

SCEPTOR is leveraging light general aviation aircraft (GA) as the performance baseline, as this is where the tradeoffs associated with DEP will be most apparent. Light GA aircraft typically have small field length requirements and associated low stall speed requirements, as they tend to be used at more than just at large commercial airports with associated long runways. Thus, their wing loading tends to be much lower than those of commercial transports. The overall low weight of these aircraft (compared to larger transport aircraft) imply that the wings of these aircraft are not overly large, even with low wing loading. As maximum lift capability increases, the wings of light GA aircraft get so small that it becomes difficult or impossible to fit the advanced high-lift device hardware such as multi-slotted flaps and actuators within the available wing volume. Finally, smaller aircraft will have lower total energy and power requirements, and the motor and battery system costs will scale with these requirements. The factors for selection of the SCEPTOR baseline aircraft included:

- Useful load (maximum net payload and fuel weight): Higher useful loads are desired as this enables the installation of larger batteries, given the fixed payload and crew requirement of 400 pounds.

- Exchange weight (defined as the weight associated with the stock gasoline engine(s) and any associated accessories that would be removed): This weight of the systems that will be removed from the retrofit aircraft translates into weight available for new systems and components. In general, electric motors tend to be lighter than the piston engines and accessories used in light GA, so a larger exchange weight for a particular power level will yield more weight available for energy storage.

- Installed power: DEP concepts should require much less power at cruise; however, the power requirements of the installed baseline engine(s) indicate the peak power requirements of the stock configuration, which should scale with the required DEP power. Furthermore, an electric version of the baseline aircraft with the baseline wing is planned as part of SCEPTOR's incremental risk reduction approach, so the installed electric motors will need to have similar peak power requirements.

- Retrofit effort: The estimated effort associated with the removal of the stock engine(s), wing, and unnecessary accessories from the retrofit aircraft will factor into cost and time required for modification. This includes the effort required to develop new non-DEP-specific components (for example, removal of the engine in a single-engine tractor configuration would also require creation of a new, faired cowl). 
- Effect of stock engine(s), wing, and accessory removal on location of center of gravity (CG): A number of components will be removed and replaced as the DEP wing and associated propulsion units and power systems are added. If the removal of the stock systems causes a large shift in the CG of the aircraft, it may be difficult or impossible to ballast or otherwise balance the aircraft with the DEP systems installed. This is generally more important than a wide CG range, since the SCEPTOR demonstrator will likely not need a large CG range (no fuel will be burned, and the aircraft will be flown by a single crewmember with a predictable weight).

- Wing carry-through location: Wing carry-through location (low-, mid-, or high-wing) effects crosswind capability and potentially impacts cost to repair damage in the event of a landing gear issue.

- Cruise speed: Aircraft designed for faster cruise capabilities will likely have lower fuselage drag, which will be much more important with the smaller DEP wing.

- Weight growth capability: The landing gear, fuselage, and other aircraft systems may be required to withstand operation at higher weights than initially certified. This may be necessary to ensure greater endurance (given that the battery system will be a significant portion of the vehicle weight).

- Manufacturer support: In-production airframes, or recently-produced airframes with solvent manufacturers, are of value as they can provide insight or potentially engineering support during modification of the airframe into the DEP configuration.

The team considered several different aircraft, including single- and twin-engine configurations, certified aircraft, experimental kitplanes, and motorgliders. Ultimately, the Tecnam P2006T, a light twin-engine training aircraft [12], was selected due to alignment with several desired factors above. The wing-mounted twin engines enable more straightforward retrofit with the DEP wing without significant changes in CG. The aircraft is available in military configurations with higher gross weights, and its service to the GA market as a training aircraft ensures that its landing gear and other systems are designed to take higher loads, should a higher gross weight be desired (the design accounts for landing loads from less experienced pilots). The wing mounts above the fuselage, enabling a high-wing configuration and associated increase in tip propeller clearance in crosswind landings. It is powered by two relatively small engines of $100 \mathrm{hp}$ peak each, and can cruise at $60 \%$ power or less. As a four-seat aircraft, there is a significant amount of fuselage volume available for SCEPTOR systems, and the available useful load is reasonable. Perhaps one of the biggest drawbacks is a relatively slow cruising speed, indicating that the fuselage may not be as clean as some of the other all-composite single engine configurations that were considered. However, the aggregate benefits of the P2006T aircraft for this retrofit application were compelling enough to merit selection by the SCEPTOR project as the baseline for retrofit to a DEP configuration.

\section{Design Approach}

The initial configuration for SCEPTOR took the form as introduced by Moore and Fredericks as the first LEAPTech (Leading Edge Asychronous Propeller Technology) concept [8], which is reproduced in Figure 1 above. The LEAPTech wing design was used, albeit with a different number of high-lift propulsors, for the SCEPTOR Mod 1 testing of the high-lift propeller concepts on a truck-mounted test platform that was raced along the dry lakebeds at NASA AFRC (Figure 4). The LEAPTech aircraft concept had a high-aspect ratio wing ( 18) with a wing loading of approximately 60 pounds per square foot. Its target cruise true airspeed was $\sim 175-200$ knots at an altitude of around $10-12,000$ feet. This was a deliberately aggressive concept to push technology limits in an attempt to showcase the potential for wing loading increase with DEP. The high aspect ratio was necessary to keep the span loading reasonable - even with the wingtip propellers, a heavily span-loaded wing would have high induced drag at cruise.

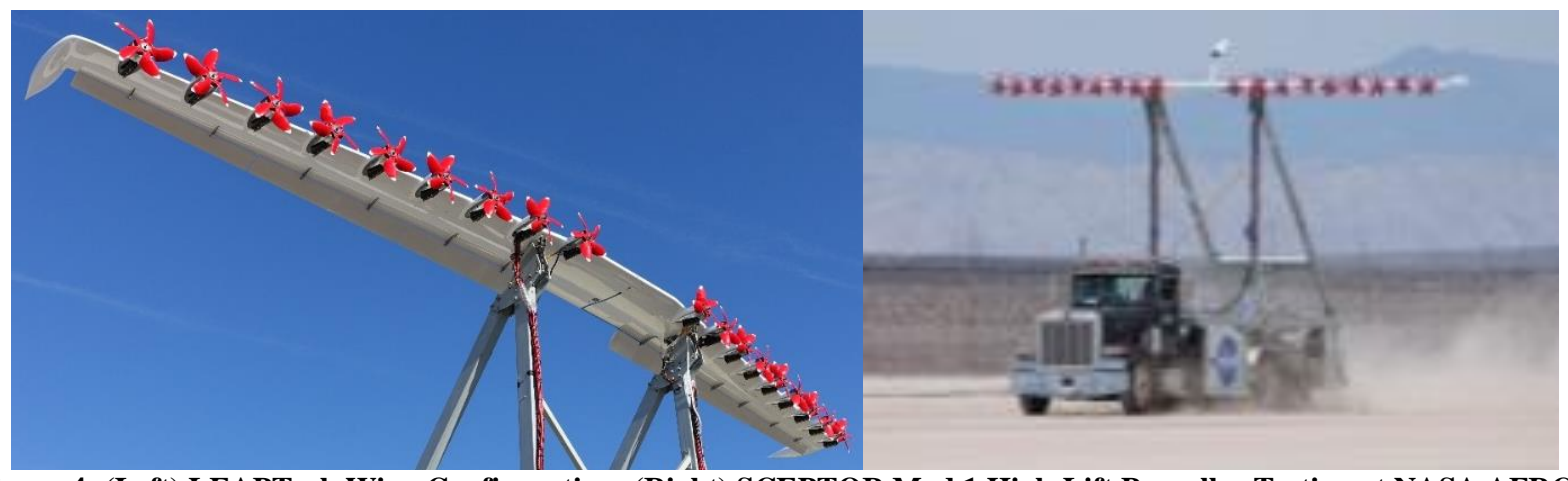

Figure 4: (Left) LEAPTech Wing Configuration; (Right) SCEPTOR Mod 1 High-Lift Propeller Testing at NASA AFRC

8

American Institute of Aeronautics and Astronautics 
The Mod 1 analysis and testing of the LEAPTech wing indicated that aggressive lift augmentation was possible with high-lift propellers. Overall, LEAPTech showed that this lift augmentation factor could exceed twice the maximum lift achievable without the high-lift propellers operating (with flaps alone). This provided the SCEPTOR team with confidence that significant increases in wing loading could occur for the flight demonstration concept. This also helped to shape the design philosophy associated with the SCEPTOR Mod 4 configuration (small wing with both wingtip cruise propellers and leading edge high-lift propellers). Ultimately, the wing would be designed for cruise performance, and for limited performance in the absence of all high-lift propellers (to ensure adequate performance in the Mod 3 configuration). That is, the wing would be sized considering only the performance with the cruise propellers operating, and then the high-lift propellers would be designed to bring the stall speed back to parity with the stall speed of the original (unmodified) aircraft as seen from the requirement in Equation (3).

\section{A. Initial Sizing}

Traditionally, new aircraft are designed to a particular mission profile often referred to as a sizing mission [13]. The sizing mission considers mission requirements such as range and payload, as well as other operational considerations such as speed, altitude, and reserve requirements. Concurrently, constraint analysis [14] helps to determine top-level aircraft parameters, such as aircraft power loading (or thrust-to-weight ratio) and wing loading. The constraint analysis and mission analysis over the sizing mission tend to use simplified, largely empirical relations to give an initial estimate of the gross weight of the aircraft. Sizing is accomplished by guessing a gross weight of the aircraft, estimating the empty weight of the aircraft through simplified relations, analyzing the mission to determine energy system weight requirements, and then iterating this analysis until convergence. If the guess for gross weight is too low, the next iteration uses a higher gross weight, and vice versa if the guess is too high.

For SCEPTOR, no hard mission requirements exist other than to attempt to demonstrate up to a $5 \mathrm{x}$ multiplier in cruise efficiency vs. a baseline aircraft. The aircraft will be limited to the test range around NASA AFRC, so no exceptional range or endurance requirements exists. For simplicity, the safety of the flight tests is based on the ability to glide to a safe landing; the dry lakebed and tens of thousands of feet of runway at the test site imply no large energy reserves for landing. For typical light aircraft cruise altitudes at typical light aircraft climb rates, this implies no more than about 20 minutes of operation. Certainly, more time is desirable, but not a necessity.

The use of a retrofit aircraft rather than a "clean sheet" design further complicates and constrains the initial sizing. Though the final aircraft will utilize a completely custom-built, DEP-enabled wing, the use of a largely stock P2006T fuselage, tail, and landing gear, along with the incremental build-up approach to testing referenced in Figure 3 , is quite constraining in terms of overall aircraft performance. Though NASA can certify aircraft to its own standards for testing on its test ranges, NASA's flight safety review process requires heavier scrutiny when evaluating experimental design features and performance characteristics vs. those that have already been evaluated via Type Certification or other processes through civil aeronautics agencies such as the FAA. Hence, to keep the flight safety certification burden low, it is best to consider keeping changes to certified aircraft operating envelopes and structural loading requirements to a minimum. For example, the Mod 2 configuration effectively helped to place a lower bound on the cruise motor power requirements. In Mod 2, the otherwise largely stock Tecnam P2006T configuration (including the stock wing) will be retrofitted with the experimental electrical power system and custom cruise electric motors (the motors that will be at the wingtips in Mod 3 and Mod 4). Hence, to have adequate performance, the cruise motors need to put out a comparable amount of power to the $74 \mathrm{~kW}$ peak normally aspirated gasoline-fueled motors on the stock aircraft. Some allowance can be made for the operating conditions, including that the normally-aspirated Rotax $912 \mathrm{~S}$ engines on the stock aircraft will not output $74 \mathrm{~kW}$ for the high density altitude conditions expected in the test range, and that the test range benefits from a very large runway and few obstacles to impede longer, flatter climbs.

The sizing of the custom wing for the SCEPTOR Mod 3 and Mod 4 flight demonstrators proceeded subject to the requirements discussed in Section II. The wing sizing environment was broken into two major efforts: design of the "cruise-sized" wing, and the design of the high-lift propeller system to enable the cruise-sized wing to meet the specified stall speed requirement. Both approaches used fast, low-order tools for rapid tradespace exploration of thousands of combinations of design variables. This paper will only briefly touch on these sizing environments. A future paper will detail the low-order integrated aerodynamic-propulsion modeling approach used for the "cruisesized" wing analysis, along with the subsequent validation of this approach to higher-order computational methods. A companion paper by Patterson et al. [15] describes the design of the high-lift propeller system, which has been built up from previous work $[6,7,16,17,18,19]$. 


\section{Cruise-Sized Wing Analysis}

The "cruise-sized" wing was developed using a series of contributing analyses for aerodynamics, propulsion, and weight estimation. A custom MATLAB $^{\circledR}$ [20] environment hosted the contributing analyses and enabled passage of critical variables and capture of key metrics. Several top-level parameters were varied using a series of tradespace exploration approaches, ranging from Design of Experiments [21] to Latin Hypercube [22] sampling. The results were visualized through a variety of plotting techniques, as well as visualized through prediction profiles generated via Response Surface Methodology [23].

No structural analysis was explicitly used during the design workflow, though structural design and aeroelastic analysis subject matter experts were consulted during the tradespace analysis to help select favorable designs. The analyses within the workflow included:

- Induced drag estimation via Athena Vortex Lattice (AVL), an open-source vortex lattice solver [24]. An AVL model of the wing and tail was generated, executed, and parsed in a series of custom MATLAB ${ }^{\circledR}$ functions. This generated induced drag estimates as well as trimmed angle of attack and stabilator positions for a sweep of input trimmed lift coefficients. Additionally, a "dummy" wing (set using AVL commands to be excluded from total force and moment output) was used to estimate the local flowfield in the vicinity of the wingtip for input into the wingtip propulsion analysis.

- Wing and tail profile and parasite drag estimation from interpolation of spreadsheet data input into the sizing environment. These data were generated prior to the tradespace exploration at various angles of attack and Reynolds numbers using XFOIL, an open-source two-dimensional panel solver with an integrated boundary layer model [25]. These included XFOIL estimates for transition from laminar to turbulent flow. The drag was aggregated via trapezoidal integration over the wing, with drag information for each section pulled from the AVL-generated lift distribution generated by spreadsheet interpolation at the AVL-estimated Reynolds number and local angle of attack.

- Nacelle parasite drag estimation for the wingtip cruise motors and high-lift motors from a simple turbulent flat plate equivalent model multiplied by a form factor. The high-lift nacelles included a higher form factor to account for additional excrescence drag due to the folded propellers, as approximated by analysis conducted by one of the authors using CFD derived from the LEAPTech configuration.

- Fuselage parasite drag estimation through spreadsheet interpolation of a component-level model from the Tecnam P2006T. This model was generated using spreadsheets developed by Patterson et al. [26] and induced drag data from VSPAero [27], calibrated to proprietary manufacturer data.

- Cruise propulsive power estimation via XROTOR, an open-source Blade Element-Momentum Theory (BEMT) solver and Minimum Induced Loss (MIL) propeller design tool [28]. An MIL propeller was designed in XROTOR and the results subsequently parsed through a series of custom MATLAB ${ }^{\circledR}$ functions. This propeller was designed with and without the radially-averaged swirl estimated from the "dummy" wing from the AVL model to help approximate the efficiency increase associated with the beneficial interaction of the propeller swirl and wingtip vortex.

- Mass property estimation via custom MATLAB ${ }^{\circledR}$ functions. This included fixed masses from the unmodified Tecnam P2006T aircraft (known from published data), as well as mass estimation for the new components. The wing weight was estimated via Raymer [29], calibrated to the stock Tecnam wing weight. The nacelles, motors, controllers, and batteries either used simple scaling estimates gathered from the LEAPTech effort or generated from component data sheets, when known. An empty weight margin (minus batteries) was added as well, and the remaining weight was assumed to be "filled" with batteries.

The airfoil design leveraged the LEAPTech efforts. LEAPTech's airfoil was identical to that used for the NASA ATLIT project [30], which utilized a NASA GA(W)-1 airfoil with a Fowler flap. Later analysis of the original LEAPTech wing showed that the $17 \%$ thick $\mathrm{GA}(\mathrm{W})-1$ airfoil suffered from high profile drag at cruise, largely because the airfoil was designed for lower cruise lift coefficients than the highly-loaded LEAPTech concept. Additionally, the small LEAPTech wing did not have much room to install the appropriate actuators for a full sliding Fowler flap. As such, the airfoil designed for the SCEPTOR Mod 3 and Mod 4 demonstrators was developed from the thinner $\mathrm{GA}(\mathrm{W})-2$ airfoil, scaled up to $15 \%$ thickness (the original $\mathrm{GA}(\mathrm{W})-2$ is a $13 \%$ thick airfoil). Some additional modifications were made to the airfoil to accommodate a single-hinge point Fowler flap, such as seen on the Cirrus SR22 and Lancair Columbia 300. As the design evolved, the airfoil design occurred outside of the sizing environment discussed above. These modifications were made to encourage laminar flow around the average design lift coefficient of the airfoil, as well as to provide better off-design performance. The airfoil design and analysis was largely centered around MSES, a multi-element Euler solver with an integrated boundary layer [31]. 
Initially, the drag of the high-lift nacelles was estimated from LEAPTech high-lift nacelle dimensions, with an estimate of 12 nacelles instead of 18 . Additional drag potential was handled using a fixed drag per dynamic pressure margin $(D / q)$ of 0.5 square feet. A fixed $D / q$ margin was used instead of a percentage of $C_{D_{0}}$ because the latter requires a reference area and would unfairly penalize larger wings.

The cruise motors were not sized to a specific performance requirement; rather, the team had initially focused on a commercial $60 \mathrm{~kW}$ continuous $/ 85 \mathrm{~kW}$ peak motor that was well suited to the project requirements as a cost-saving measure. This power level would be sufficient for operation with the stock Tecnam aircraft in the Mod 2 configuration, and makes it unlikely for the aircraft to exceed the maximum speed of the aircraft (at least in level flight) for the Mod 3 and Mod 4 configurations. This precludes the need to recertify or test unmodified components for higher speeds (such as the horizontal and vertical tail). Later iterations forced SCEPTOR to drop the planned commercial motor, but the power requirements of $60 \mathrm{~kW}$ continuous remained due to design heritage.

Early in the project, the team needed to determine if the cruise propellers at the wingtips should adopt a fixedpitch configuration or allow for variable-pitch operation. In general, variable pitch propellers enable a wider range of operation than fixed pitch, yet fixed pitch propellers tend to be less expensive, less complex, and lighter. McDonald recently showed that variable pitch propellers tend to continue to offer efficiency advantages over fixedpitch propellers when coupled with electric motors, despite the much wider efficiency islands electric motors enjoy across a range of RPM [32]. Additional uncertainty in the performance model of the aircraft and of the electric motors (particularly after the switch to a custom cruise electric motor) pushed the team towards a variable-pitch solution. Though the team would later select a commercially available propeller, initial estimates for performance and swirl were derived from an XROTOR MIL propeller sized to absorb $85 \mathrm{~kW}$ at the selected cruise point, representing a burst top-of-climb capability.

\section{Tradespace Exploration}

The "cruise-sized" wing tradespace exploration went through three major cycles. Initially, the wing tradespace included variations for a two-segment wing, with an inner and outer section, and a cruise propeller design. For the first and second major cycles, the parameters for the wings and cruise propellers were sampled using Latin Hypercubes [22]. Each wing sample generated an AVL trimmed $C_{L}$ sweep as described above, and each cruise propeller parameter sample generated a MIL propeller design in XROTOR. Each combination of wing and propeller data were then evaluated to determine the range efficiency multiplier $R_{\eta}$ per Equation (1) and a total range parameter $R_{T}$. The latter parameter was simply determined by

$$
R_{T}=\left(m_{\text {batt }} e_{\text {batt }} / P_{\text {cruise }}\right) V_{\text {cruise }}
$$

where $m_{\text {batt }}$ is the mass of the battery, and $e_{\text {batt }}$ is the specific energy capacity assumed for the battery. Note that this is really an upper bound on aircraft range, since there will be additional inefficiencies associated with takeoff, climb, and reserve requirements. It simply gives a "range-like" parameter to judge different designs.

The primary purpose of tradespace exploration is discovery, not design. The analysis approach highlighted above was selected deliberately because of its exceptionally low execution time to evaluate a single design, allowing for thousands to millions of combinations of design variables to be evaluated on a simple laptop computer or small cluster, with runs rarely lasting more than overnight. A total of three major tradespace iterations were made over the course of the initial wing sizing, evaluating millions of combinations of wing and cruise propeller designs. The tradespace evolution is shown in Table 1.

Table 1: Wing/Cruise Propeller Tradepsace Exploration Epochs

\begin{tabular}{llll} 
Epoch & Exploration 1 & Exploration 2 & Exploration 3 \\
\hline Wing variables & 7 & 4 & 3 \\
Wing sampling method & Latin Hypercube & Latin Hypercube & 6 level full factorial \\
\hline Total unique wings & 1000 & 500 & 216 \\
\hline Propeller variables & 5 & 4 & 4 \\
Propeller sampling method & Latin Hypercube* & Latin Hypercube* & Latin Hypercube* \\
\hline Total unique propellers & 200 & 200 & 200 \\
\hline Gross weight & $2700,3000,3400$ pounds & $2700,3000,3400$ pounds & $2700,3000,3400$ pounds \\
\hline Cruise speed & $150,175,200 \mathrm{KTAS}$ & $135,150,175 \mathrm{KTAS}$ & $135,150,175 \mathrm{KTAS}$ \\
\hline Total combinations & $1.8 \mathrm{M}$ & $900 \mathrm{k}$ & $388 \mathrm{k}$
\end{tabular}

*One variable was discrete (number of blades), so a lower-variable LHC design was duplicated for each discrete variable setting. 
The designs were filtered by sorting the top designs with respect to the efficiency multiplier for each of the unique combinations of gross weight and cruise speed. When plotted versus the range parameter, this helped to identify designs that could provide some meaningful data at cruise (those with a longer ranger parameter), while at the same time identifying the effect of increased weight (and therefore battery energy) and higher cruise speed. Initially, the designs pushed towards large wingspans and high propeller diameters, as would be expected for greater efficiency improvements. However, this could constrain the bank angle constraint in a crosswind landing as discussed earlier. Hence, the results were filtered to rule out designs that did not give sufficient margin for some crosswind landing. Figure 5 shows the filtered tradespace results that allow for a minimum of 10 degrees of bank during a crosswind landing.

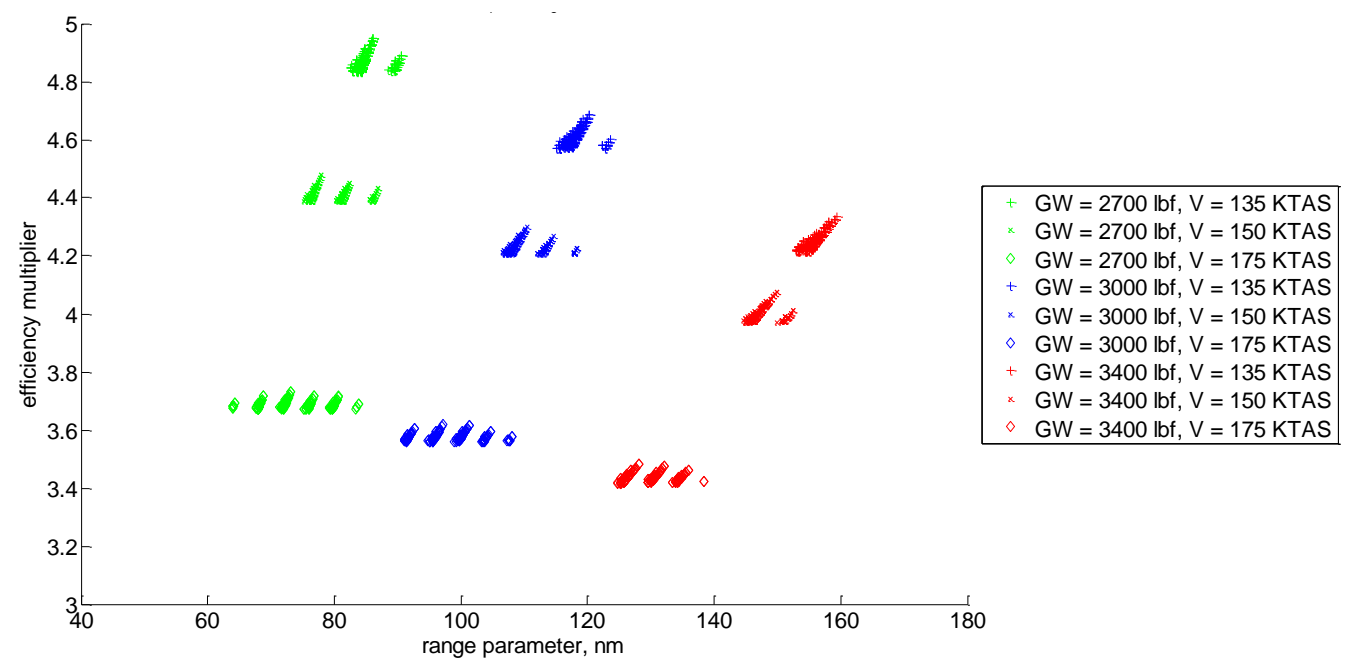

Figure 5: Wing/Cruise Propeller Tradespace for Top 200 Designs in Exploration Epoch 3 with Bank Angle Constraint

The tradespace results showed that it was possible to meet the efficiency multipliers per the project goals (minimum 3.5x improvement, goal of 5.0x), but did not necessarily give insight into the effect of the wing and propeller design variables on the performance of the aircraft. This could be inferred from a matrix of twodimensional scatterplots, where the variables of the top designs were plotted against each other. Large spreads tend to indicate that little correlation exists between the design variables for the top designs, whereas clusters indicate preferred settings. Figure 6 shows one such scatterplot matrix developed in Exploration 3. Note that wing loading tended to cluster to nominal values, and cruise propeller diameter pushed towards the upper limits. Also note how some variables, such as propeller design lift coefficient, had little correlation - effectively, when conducting a MIL design of a propeller, it is possible to create an efficient on-design configuration for any manner of design lift coefficients. Instead, off-design behavior tends to push propeller design lift coefficient $[7,15]$.

The thousands to millions of variables within the tradespace provided a rich dataset for further interrogation and visualization through Response Surface Methodology. Figure 7 shows the response surface prediction profile generated by MATLAB's rstool [33], with a number of additional metrics and design variables. The metrics, from top to bottom, are crosswind bank angle, minimum power required, specific excess power, power-off rate of descent, glide ratio, range parameter, and efficiency multiplier. The variables, from left to right, are aspect ratio, wing loading, taper ratio, washout, propeller tip speed, and propeller diameter. As seen from the profiler, some variables have a strong effect on the design, and others tend to have little effect and can be set via other constraints. For example, washout had little effect on nominal aircraft performance, but does have an effect on the lift distribution (particularly near stall). Hence, washout was set by first selecting an appropriate wing planform, and then tailoring the washout to ensure little tendency for tip stalls by scrutinizing the lift distribution.

The prediction profiler provided the team with a rich environment to quickly game various scenarios, and enabled other subject matter experts (such as test pilots, aeroelasticians, and structural analysts) to see the effect of "de-tuning" the optimal design to meet what may otherwise be considered subjective constraints. One of the main drawbacks of design optimization using low-order tools is that an optimizer can exploit weaknesses in the model. By using the tradespace-centered approach, the team could select a "satisificing" design [34] that, in the opinion of the subject matter experts, would also be passable with respect to the unmolded phenomena. 


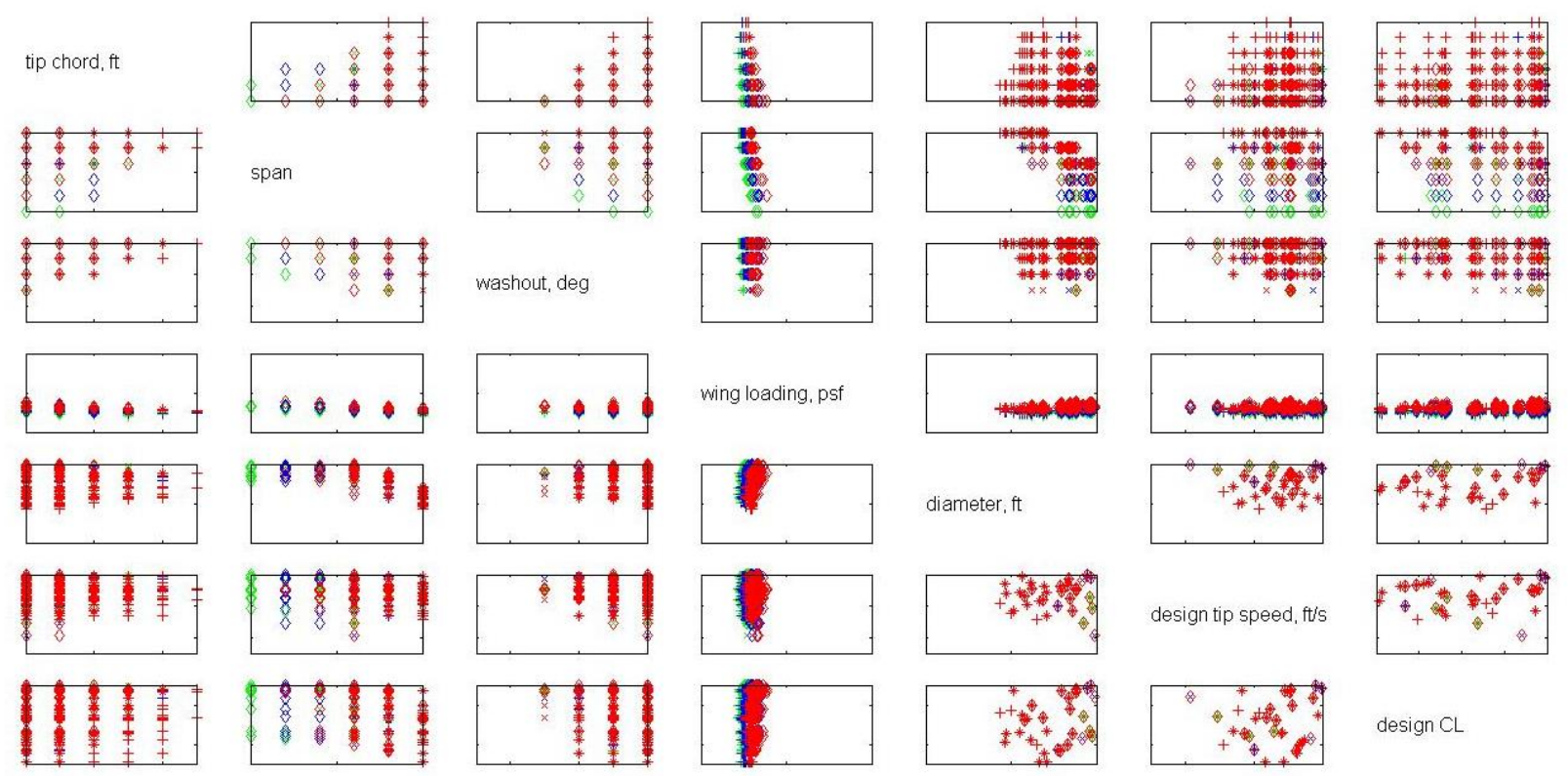

Figure 6: Scatterplot Matrix of Design Variables for Top 200 Designs in Exploration Epoch 3
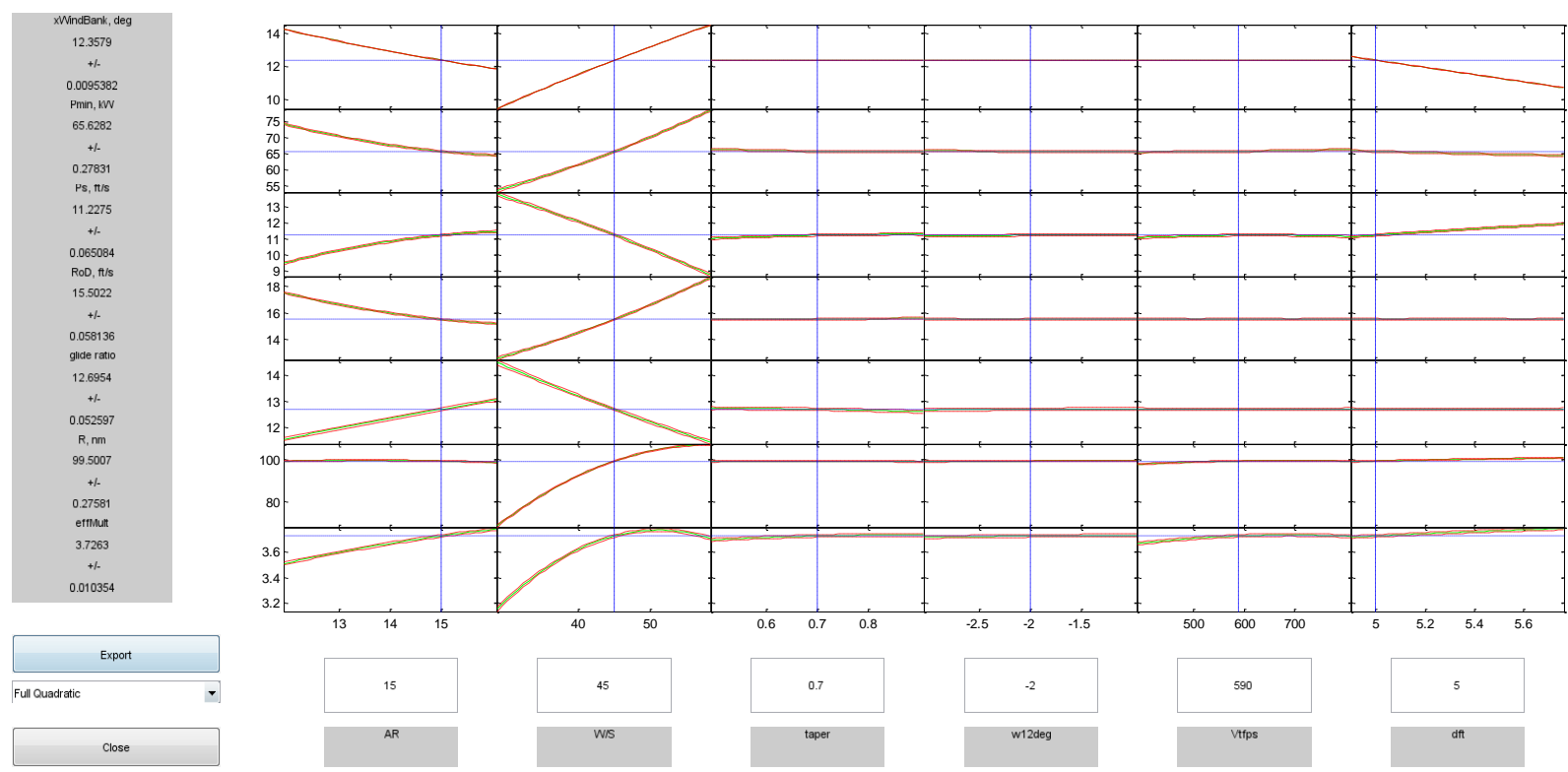

Figure 7: Response Surface Prediction Profile (generated via MATLAB ${ }^{\circledR}$ rstool [33])

\section{High-Lift Propeller Selection}

The selection of a cruise-sized wing enables the design of the associated high-lift propeller system, one of the major benefits of DEP technology. A number of factors need to be considered: the diameter of the propellers, the angle and offset of the propellers with respect to the wing, and the acoustic signature of these propellers. The scope of these discussions is far too broad for this single paper. Rather, the reader is referred to discussions by Borer et al. [7], Stoll et al. [35], and most recently the companion paper by Patterson et al. [15]. Patterson's recent dissertation [36] provides an in-depth discussion of the multitude of effects and models necessary to select an appropriate highlift propeller system, using the SCEPTOR flight demonstrator as its example. 


\section{SCEPTOR Design and Performance Characteristics}

The rapid tradespace exploration tools developed for the SCEPTOR flight demonstrator enabled the creation of multiple revisions used to feed more detailed preliminary design analysis. As discussed above, this enabled near real-time creation of new designs while collecting subject matter feedback. The tradespace iterations indicated that a target cruise speed of 150 knots true airspeed (KTAS) and a gross weight of 3,000 pounds would enable a solution that could meet the appropriate efficiency multiplier while also giving a comfortable mass margin for a larger energy storage system. The stock Tecnam P2006T has a gross weight of up to 2,712 pounds, depending on configuration. The higher weight was of little concern, as the FAA grants special flight permits to certified aircraft with some restrictions on the aircraft operations that may not require any physical alteration of the aircraft [37]. This also fixed the DEP stall speed requirement discussed in Equation (3) to approximately 58 knots calibrated airspeed (KCAS), which ultimately enabled a more straightforward design procedures for the high-lift propeller system.

\section{A. Design Revisions}

A number of different revisions were broadcast to the larger team as the design proceeded to its Preliminary Design Review. Table 2 shows the salient characteristics of several different revisions alongside a comparison to the stock Tecnam P2006T. Overall, it is apparent that there is a significant reduction in wing area, and increase in aspect ratio, as compared to the stock aircraft.

Table 2: SCEPTOR Design History Prior to Preliminary Design Review

\begin{tabular}{|c|c|c|c|c|}
\hline Model & P2006T (stock) & Rev12 & Rev 20 & \\
\hline Span, ft & 37.4 & 33.0 & 29.2 & 31.6 \\
\hline Planform area, $\mathrm{ft}^{2}$ & 158.9 & 56.9 & 57.5 & 66.7 \\
\hline Wing loading, lbf/ft $\mathbf{2}^{2}$ & 17.1 & 52.7 & 52.2 & 45.0 \\
\hline Aspect ratio & 8.8 & 19.1 & 14.8 & 15.0 \\
\hline Root chord, ft & 4.57 & 2.25 & 1.97 & 2.48 \\
\hline Tip chord, ft & 2.90 & 1.20 & 1.97 & 1.74 \\
\hline Leading edge sweep, deg & 0.0 & 5.0 & 7.5 & 1.9 \\
\hline Cruise propeller diameter, $\mathbf{f t}$ & 5.84 & 4.70 & 5.74 & 5.00 \\
\hline Cruise propeller speed at cruise, RPM & 2250 & 2470 & 1500 & 2250 \\
\hline Number of high-lift propellers & 0 & 8 & 10 & 12 \\
\hline
\end{tabular}

In the first major revision of the design, the wing was highly loaded near the peak efficiency multiplier at nearly $53 \mathrm{lbf} / \mathrm{ft}^{2}$. It also had a very high aspect ratio, which, combined with the low wing area and higher taper ratio, resulted in a very small chord at both the wing root and wingtip. This design also had a mild leading edge sweep to provide the high-lift propeller discs some axial separation. This first revision also had only eight high-lift propellers, due to concerns associated with reduction in the lift augmentation effect associated with the height of the blown slipstream [18]. Unfortunately, the larger diameter of the high-lift propellers introduced a large excess thrust in the approach configuration, which could make approach to landing at low speed quite difficult.

Other issues with the first revision were voiced by the SCEPTOR structures team with respect to the very small wingtip chord. The wingtip needs to house the structural attachment for the wingtip-mounted cruise motors, and a mere 1.2 feet chord and an airfoil thickness ratio of $15 \%$ resulted in a maximum wingtip thickness of slightly more than two inches. Given these concerns, the team briefly considered the Rev2.0 configuration, which maintained a small, highly loaded wing, but with a reduced aspect ratio and no taper to enable a larger tip chord. This brought up significant concerns with the wing root spar depth and bending moment, so the team quickly moved on to its final configuration prior to PDR, Rev3.

The Rev3 configuration incorporated several changes. First, the wing loading was reduced slightly to enable a larger wing, though the efficiency multiplier suffered somewhat (approximately a $4 \%$ reduction). Next, the wing sweep was essentially eliminated, favoring zero sweep at the $70 \%$ chord line - right at the estimated location of the aft spar and just forward of the aileron and flap hinge line. The lack of leading edge sweep required the high-lift 
propellers to be "staggered" fore and aft to ensure adequate axial separation between the adjacent propeller discs. As the Rev3 analysis continued, the high-lift propeller lift augmentation models matured enough to allow the team to gain more confidence to move towards more propellers, moving up to 12.

Throughout all of these revisions, an interesting trade occurred between wing span, cruise (tip) propeller diameter, wing span loading, and propeller tip speed. Namely, the drag reduction benefits of wingtip-mounted propellers are greater for higher span loading, smaller propeller diameter-to-wingspan ratio, and higher flight velocity-to-propeller tip speed ratio [10]. This would seemingly push towards smaller, slower-turning propellers, on smaller spans (to increase span loading). However, in general, propulsive efficiency will be increased for isolated propellers at higher propeller tip speed (though below sonic velocity) and larger diameters, and aerodynamic efficiency will be increased for lower span loading. Overall, the trades tended to push towards the larger spans and larger propeller diameters, though this is ultimately limited by aircraft crosswind angle.

\section{B. SCEPTOR 4.0}

SCEPTOR held its Preliminary Design Review in November 2015, and is rapidly moving towards its Critical Design Review, which will effectively "lock" the configuration. The major design features of the latest SCEPTOR revision, dubbed SCEPTOR 4.0, is shown in Figure 8. This is very similar to the Rev3.3 configuration used for PDR; indeed, the major specifications shown for Rev3.3 in Table 2 are identical for the SCEPTOR 4.0 configuration. Some changes have necessarily been incorporated as the design has progressed, particularly with respect to the cruise motor and propeller. The wingtip cruise nacelle is larger in diameter to accommodate the latest design of the air-cooled custom Joby outrunner motor, and the tip nacelle is longer to accommodate motor controllers and data acquisition units. Also, the propeller disc has been translated aft from the Rev3.3 configuration to reduce structural issues with integrating the tip nacelle into the wing structure.

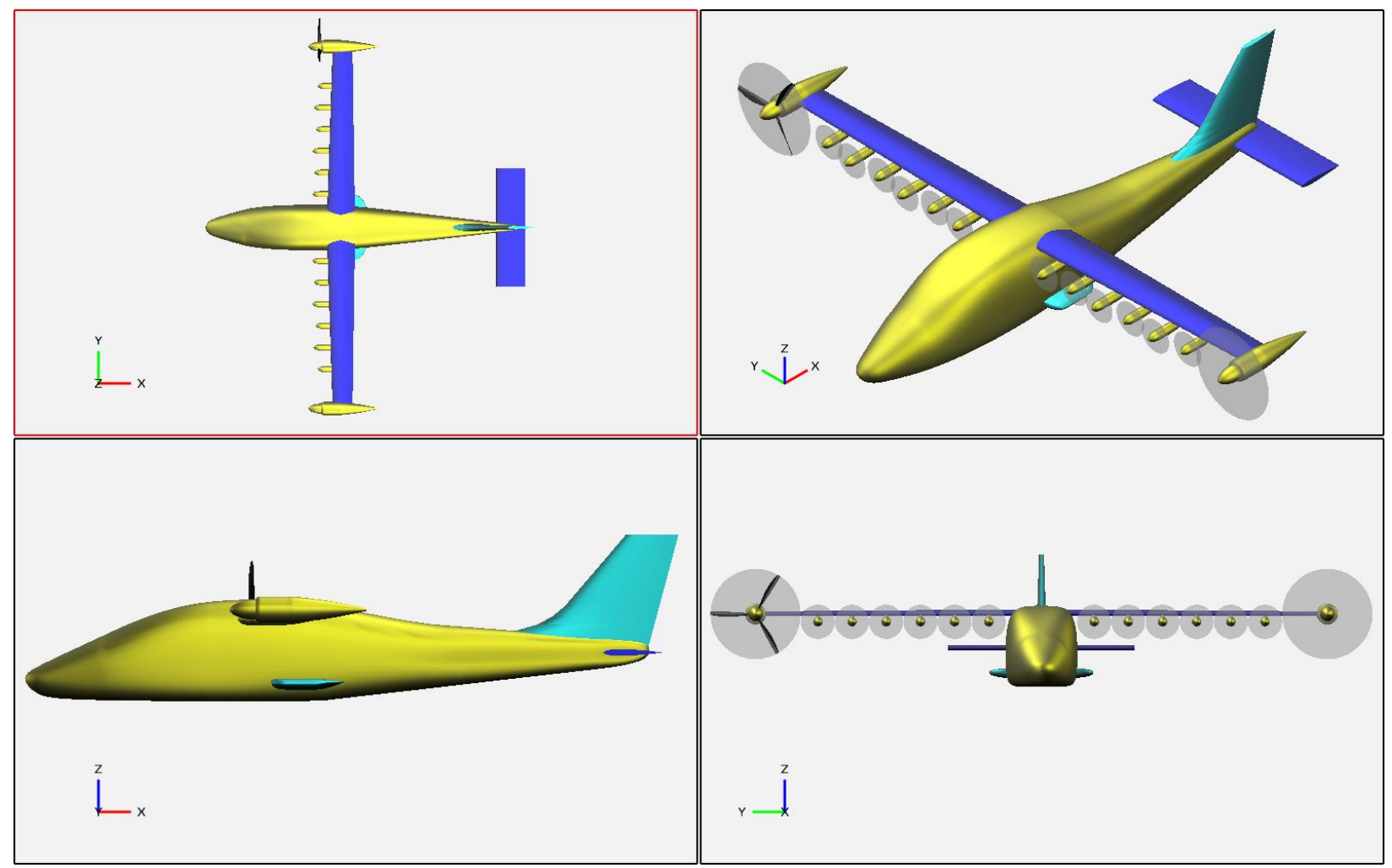

Figure 8: SCEPTOR 4.0 Configuration - Generated with OpenVSP [38]

Other, more subtle changes include additional detail related to the motor cooling. The custom Joby cruise motors and controllers are air-cooled, and a radial inlet ensures adequate cooling flow to the motor stator and magnets. Nacelle cooling design is discussed in a companion paper by Dubois et al. [39]. Additional cooling flow is directed to the dual motor controllers located behind the motor. The nacelle diameter was increased due to selection of a commercially-available propeller and spinner with a larger hub size than initially assumed. The team selected MT propeller's MTV-7A-152/64 propeller [40], which uses composite-wrapped wood core blades and an electrically- 
actuated constant speed blade pitch controller. The use of a commercial propeller cost an estimated 1-2\% in cruise efficiency vs. the custom-designed propeller from Rev3.3, but comes reduces development cost and risk (for example, the MIL propeller design from Rev3.3 did not include structural or vibrational constraints, whereas the commercial propeller selected has an FAA Type Certificate and has already been subjected to rigorous tests).

Other notable features include the pronounced "stagger" of the high-lift propeller discs. The small amount of leading edge wing sweep results in axial separations between adjacent high-lift propellers that is far too small. Typical guidance suggests that propeller blades should have a five-degree offset from adjacent propellers to mitigate the probability of cascading failures. Additionally, the blades of the high-lift propellers must be far enough ahead of the wing to enable folding. Though the detailed design of the folding propellers and high-lift nacelles is ongoing, the initial high-lift nacelle locations ensured that at least one blade radius (minus hub radius) plus a small margin.

The high-lift nacelles themselves are placed on pylons under the wing. The exact offset is to be determined, though the initial offset was found using a procedure similar to that outlined by Borer et al. [7]. Recent, more detailed computational studies by the authors have indicated that longer pylons may yield greater high lift benefits; however, this may ultimately be limited by aeroelastic tendencies. The use of pylons, rather than integrated nacelles as on the LEAPTech concept, enables pylon height and nacelle design trades to continue while the wing primary structure is designed. It also helps to reduce the influence of the high-lift nacelles on the aerodynamics of the wing, though there is still some impact.

\section{Performance}

One of the fundamental benefits of DEP technology is the shifting of the maximum $L / D$ ratio to higher velocities, as discussed earlier. The smaller wing enabled by the higher maximum lift capability also serves to reduce wing wetted area, which can increase $L / D$ for a given fuselage size (though the increased parasite drag of the high-lift nacelles, along with the increase in profile drag due to operating a high lift coefficients, can offset this effect). Finally, the wingtip-mounted cruise propellers can reduce induced drag, further increasing $L / D$. The estimated $L / D$ of the SCEPTOR 4.0 configuration vs. altitude and velocity is given in Figure 9.
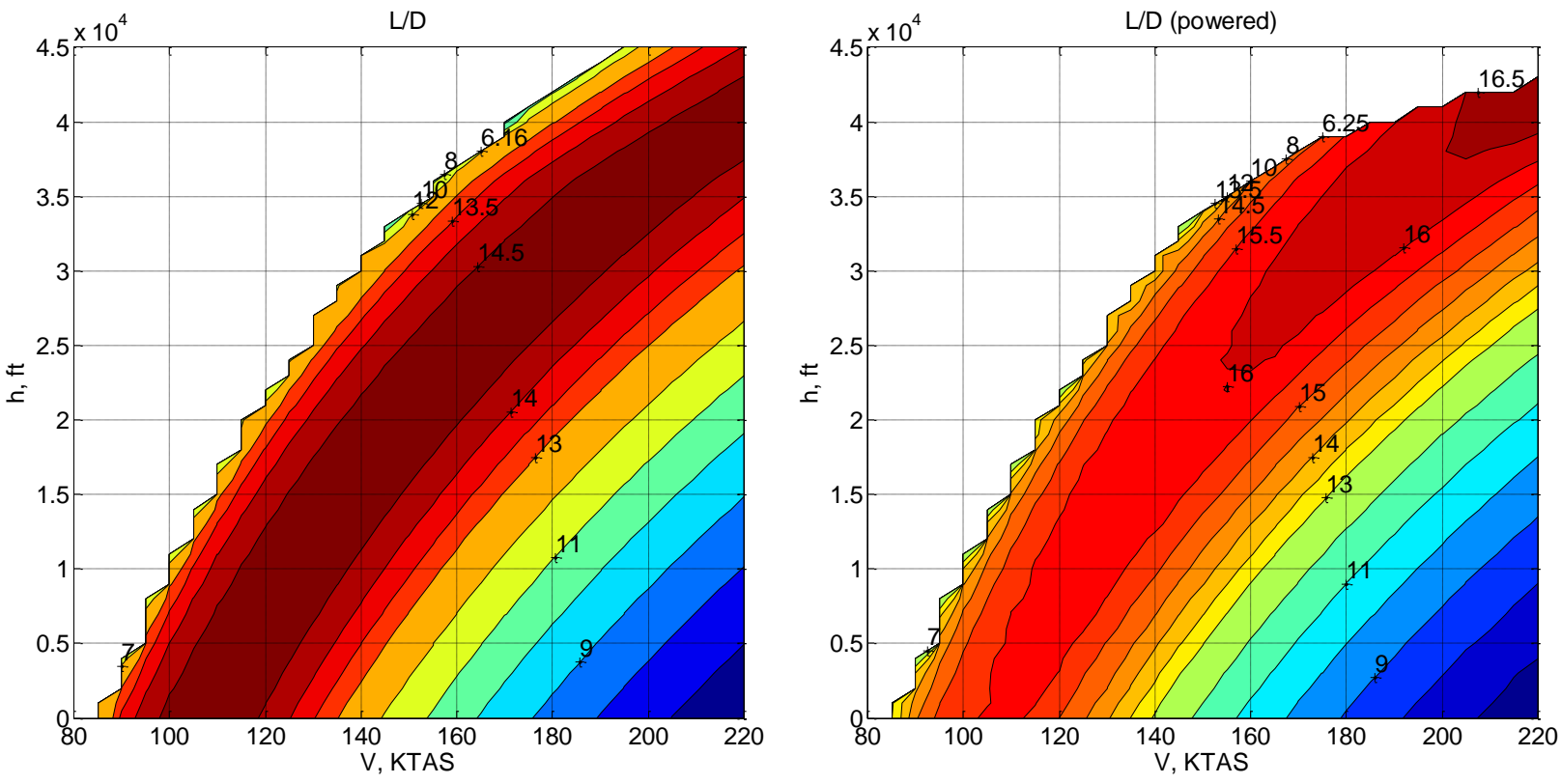

Figure 9: SCEPTOR 4.0 L/D Performance - (Left) Power off, (Right) Cruise Propellers at Power for Level Flight

One immediately apparent feature is the increase in $L / D$ with the tip propellers operating throughout much of the flight envelope with power for level flight. The wingtip propeller effect tends to add about 5-10\% to the $L / D$ as it is modeled here; however, more detailed CFD studies by the authors indicates that the grossly simplified AVLXROTOR tip propeller modeling approach is likely underpredicting this effect by a fair amount. Additionally, the speed for maximum $L / D$ is increased over the stock Tecnam P2006T configuration. The best glide speed is published in the P2006T Pilot Operating Handbook as 85 knots calibrated airspeed (KCAS) [41], which is typically near the velocity for maximum $L / D$ (if not slightly higher, for operational liability). The maximum power-off $L / D$ for SCEPTOR 4.0 occurs around $110 \mathrm{KCAS}$, indicating the effect of wing loading increase. 
The rate of climb and associated climb gradient are shown in Figure 10. These plots assume the cruise propellers at the wingtips are operating at maximum continuous power (MCP, $60 \mathrm{~kW}$ each) with no thrust contribution from the high-lift propellers. Such a situation applies to the entire flight envelope of the Mod 3 demonstrator, and is also applicable for the Mod 4 demonstrator shortly after transition into cruise climb. While the climb performance is not stellar, it exceeds the project-imposed climb gradient requirements introduced earlier.
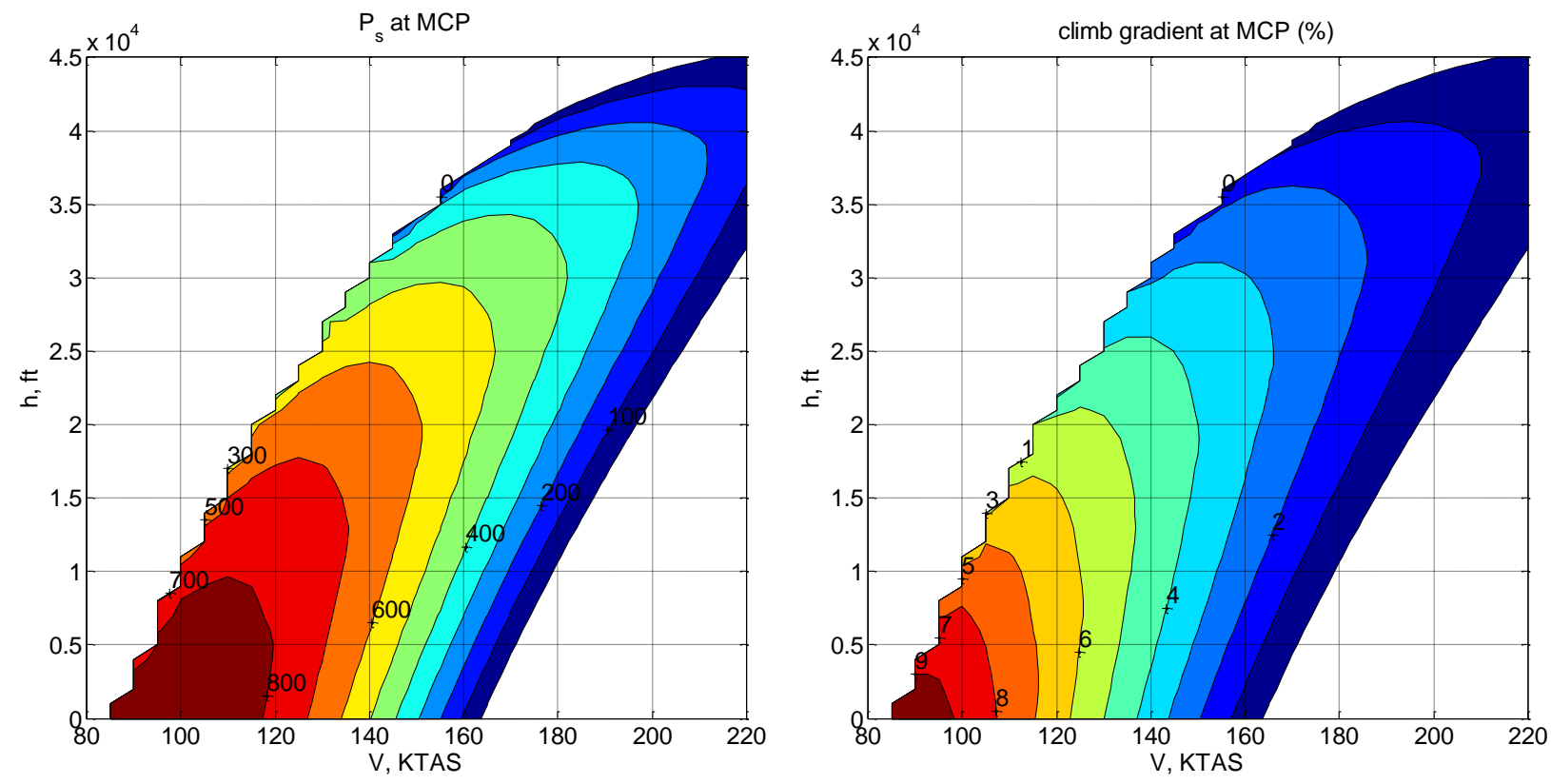

Figure 10: SCEPTOR 4.0 Cruise Configuration Climb Performance (Left) Rate of Climb (ft/min), (Right) Climb Gradient (\%)

One interesting note about the climb performance charts is the lack of power lapse with altitude. One of the benefits of electric motors is that they act the same as a turbonormalized powerplant - they do not experience power lapse as altitude is increased (conversely, they also do not get any benefit from ram air). The propeller itself lapses, hence it eventually cannot produce enough thrust to keep the aircraft airborne. Still, this highlights one of the benefits of electric motors, should the energy storage problem be alleviated - the aircraft are still capable of climbing at altitudes much higher than frequented by current light aircraft with normally aspirated engines.

Related to this, the power required for level flight and the estimated efficiency multiplier vs. the stock Tecnam P2006T are given in Figure 11. The P2006T efficiency data is extrapolated from a simple model based on the fuel flow rates published in the operating handbook [41]. Certainly, there are airspeed and altitude combinations that are inappropriate for the stock aircraft to operate shown in this figure, so it is to be interpreted accordingly. Also, some discontinuities are seen in the contours where there were some difficulties extrapolating the P2006T drag data from the pilot operating handbook. The purpose of the SCEPTOR Mod 1 flights with the stock aircraft were to determine the exact efficiency multiplier; however, data validation is ongoing, so only the operating handbook data is shown.

The cruise performance of SCEPTOR shows once again that the aircraft is capable of flying quite high and fast, showing the potential for technologies such as DEP to enable greater mobility when onboard electricity storage (or onboard electricity generation from fuels) allows for greater climb durations. When compared on an altitude- and airspeed-basis, the SCEPTOR 4.0 demonstrator is capable of an efficiency multiplier of over 5.0, and approximately 4.8 at the selected cruise point of 150 KTAS and 8,000 feet altitude. While more work needs to be accomplished with higher-order modeling and testing, this is a very encouraging development. 

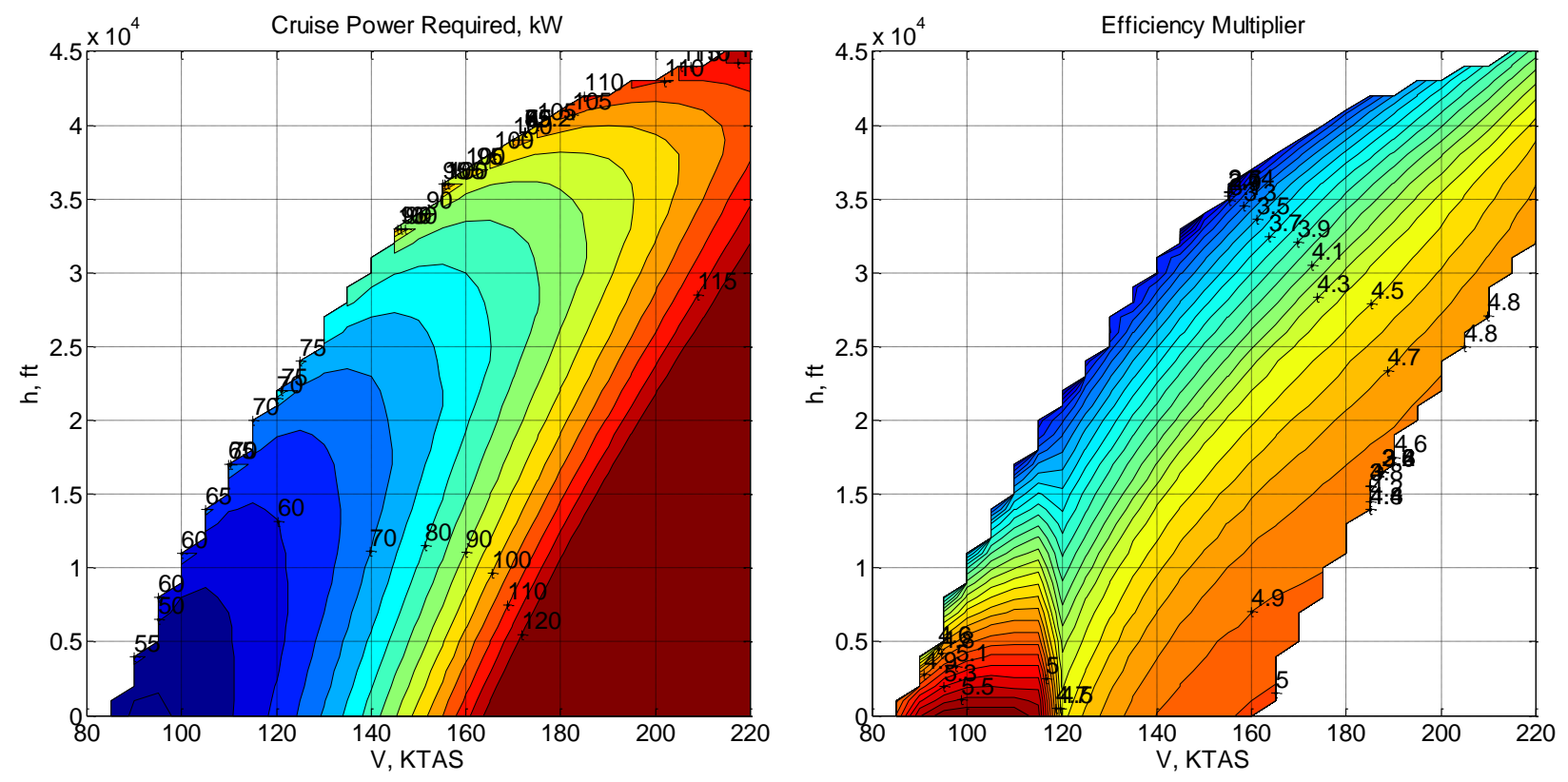

Figure 11: SCEPTOR 4.0 Cruise Performance - (Left) Power Required for Level Flight $(\mathrm{kW})$, (Right) Efficiency Multiplier

\section{Future Revisions}

Much of the future work focuses on the development of the high-lift propeller system. As mentioned earlier, the placement of the high-lift propellers on pylons under the wings allows for more detailed wing design to commence while more detailed work on the high-lift propellers and nacelles continues. The design techniques referenced earlier in this paper do indicate that high-lift propeller designs are available to meet the DEP-enabled stall speed of 58 KCAS, though the approaches used to create these propellers are not yet fully integrated with high-lift nacelle design. This integration is essential as it allows for the concurrent exploration of nacelle shapes for cruise flight that encourage low-drag propeller folding, but also do not severely degrade the propeller performance.

Another major consideration for high-lift propellers is the approach profile and high-lift motor control scheme. Patterson [36] explores this from the point of view of safety margin over wind shear, which is the primary reason that approaches to landing do not occur right above stall speed, but at some comfortable margin above it. These profiles will need to be investigated with more rigorous low-speed drag models, as well as with input from the highlift motor control development team.

Once the high-lift propeller system is better defined, the effects of these high-lift propellers on the overall performance metrics (largely, rate of climb and rate of descent) need to be considered. In addition, the effect of offnominal events, such as the failure of one of the high-lift propellers during critical operations, will be modeled. This is in addition to the modeling of the failure of the cruise propellers (this is ongoing and is a recognized hazard due to the potential for large motor-out yawing moments).

\section{Conclusions}

Airborne electric propulsion is a topic of increasing interest in the aviation community, primarily due to the potential for a large efficiency advantage over existing combustion-based powerplants, which can exceed a factor of three for small aircraft designs. As onboard electricity storage or generation improves, electric propulsion offers compelling advantages. One of the most interesting features of airborne electric propulsion is how the scaling and efficiency differences from traditional combustion-based propulsors enable far different airframe-propulsion integration, in particular allowing for beneficial coupling between these two traditionally independent disciplines.

NASA's SCEPTOR project aims to show up to a fivefold improvement in cruise efficiency through flight demonstration of a retrofit general aviation aircraft. This large increase is due to efficient electric propulsion and airframe/propulsion integration through Distributed Electric Propulsion technology. The SCEPTOR flight demonstrations culminate with a configuration that includes two different types of DEP: a dozen small high-lift motors and propellers to accelerate the flow over a wing at low speeds, and two larger cruise motors and propellers 
co-located with each wingtip to provide primary propulsive power. The high-lift propellers enable a highly-loaded wing that is more efficient at cruise without sacrificing low-speed performance, and the wingtip cruise propellers enable increased cruise efficiency through beneficial interaction with the wingtip vortex, resulting in less propulsive power required for a given flight velocity.

This paper presents the driving requirements and desires for SCEPTOR, the criteria used by the team to select the candidate aircraft for retrofit, and details the tradespace exploration approach used to define this complex configuration. The SCEPTOR team created a rapid design framework to quickly cull through millions of combinations of candidate wing and propeller parameter combinations to build intuition into the salient trades related to the design of the new aircraft components. This design environment allowed for communication with subject matter experts within the team to identify design features that would be favorable but are otherwise unmodeled by the parametric design tools. As the design progressed, and other disciplinary teams within SCEPTOR were able to mature their analyses and validate assumptions, these same tools were used to evolve the SCEPTOR demonstrator configuration through three major revisions. The current demonstrator design, dubbed SCEPTOR 4.0, includes the latest details gathered by the disciplinary teams and related development efforts. The SCEPTOR 4.0 performance indicates that the aircraft will nearly meet its stretch goal of efficiency improvement: the latest estimates show an efficiency multiplier of approximately $4.8 \mathrm{x}$ better than the stock aircraft at the selected cruise point of 150 knots true airspeed and 8,000 feet altitude.

The SCEPTOR 4.0 configuration will continue to evolve as the SCEPTOR project proceeds to its next major milestone, the Critical Design Review. In particular, the high-lift propeller system will be further refined to ensure the aircraft can meet its low-speed performance goals, without additional sacrifices to cruise performance. More than a paper study, this aircraft ultimately will fly in several demonstrations in the coming years as NASA's next "X-Plane" to showcase the benefits of electric aircraft and Distributed Electric Propulsion Technology.

\section{Acknowledgments}

This work was funded under the NASA Convergent Aeronautics Solutions Project of the Transformative Aeronautics Concepts Program. The authors would like to thank the entire SCEPTOR team, which includes three NASA research centers (Armstrong Flight Research Center, Glenn Research Center, and Langley Research Center) and three major contractors (Empirical Systems Aerospace, Inc., Joby Aviation, and Xperimental LLC). The DEP concept aircraft in Figure 1 was developed by Bill Fredericks, Andy Hahn, and Mark Moore of NASA Langley.

\section{References}

1. M. Moore, K. Goodrich, J. Viken, J. Smith, B. Fredericks, T. Trani, J. Barraclough, B. German, M. Patterson, "High-Speed Mobility through On-Demand Aviation," AIAA 2013-4373, Aviation Technology, Integration, and Operations Conference, Los Angeles, CA, August 2013.

2. Airbus Group, "Airbus E-Fan: the future of electric aircraft," http://www.airbusgroup.com/int/en/innovation-environment/efan-the-electric-plane.html, accessed 13 May 2015.

3. M. Grady, "Pipistrel Introduces Alpha Electro," http://www.avweb.com/avwebflash/news/Pipistrel-Introduces-AlphaElectro-223852-1.html, accessed 13 May 2015.

4. N. K. Borer, C. L. Nickol, F. P. Jones, R. J. Yasky, K. Woodham, C. M. Cagle, J. S. Fell, B. L. Litherland, P. L. Loyselle, A. J. Provenza, L. W. Kohlman, A. G. Samuel, "Overcoming the Adoption Barrier to Electric Flight," AIAA SciTech 2016, San Diego, California, January 2016.

5. R. A. McDonald, "Establishing Mission Requirements Based on Consideration of Aircraft Operations," Journal of Aircraft, 50(3) 741:751, 2013.

6. N. K. Borer, M. D. Moore, A. R. Turnbull, "Tradespace Exploration of Distributed Propulsors for Advanced On-Demand Mobility Concepts," AIAA-2014-2850, AIAA Aviation, Atlanta, GA, June 2014.

7. N. K. Borer, M. D. Moore, "Integrated Propeller-Wing Design Exploration for Distributed Propulsion Concepts," AIAA 2015-1672, AIAA SciTech, Kissimmee, FL, 5-9 January 2015.

8. M. Moore and B. Fredericks, "Misconceptions of Electric Propulsion Aircraft and their Emergent Aviation Markets," AIAA 2014-0535, 52nd Aerospace Sciences Meeting, National Harbor, MD, January 2014.

9. G. Warwick, "NASA’s Electric-Propulsion Wing Test Helps Shape Next X-Plane,” Aviation Week \& Space Technology, 24 August 2015.

10. L. R. Miranda, J. E. Brennan, “Aerodynamic Effects of Wingtip-Mounted Propellers and Turbines,” AIAA-86-1802, 1986.

11. Code of Federal Regulations, Title 14, "Aeronautics and Space," Part 23, "Airworthiness Standards: Normal, Utility, Acrobatic, and Commuter Category Airplanes," Amdt. 62. Available at www.ecfr.gov, accessed 20 April 2016.

12. “P2006T - Tecnam," http://www.tecnam.com/aircraft/p2006t/, accessed 10 May 2016.

13. D. N. Mavris, N. K. Borer, "Development of a Multi-Mission Sizing Methodology Applied to the Common Support Aircraft," SAE-2001-01-3014, Aerospace Congress and Exhibition, Seattle, WA, 11-13 September 2001 
14. J. D. Mattingly, W. H. Heiser, D. T. Pratt, Aircraft Engine Design, Second Edition, American Institute of Aeronautics and Astronautics, Reston, Virginia, 2002.

15. M. D. Patterson, N. K. Borer, A. M. Stoll, J. M. Derlaga, "High-Lift Propeller Design for NASA's SCEPTOR Distributed Electric Propulsion Flight Demonstrator," AIAA Aviation 2016, Washington, D.C., June 2016.

16. M. D. Patterson, M. J. Daskilewicz, B. J. German. "Conceptual Design of Electric Aircraft with Distributed Propellers: Multidisciplinary Analysis Needs and Aerodynamic Modeling Development," AIAA 2014-0534, AIAA SciTech 2014.

17. M. D. Patterson, B. J. German. "Wing Aerodynamic Analysis Incorporating One-Way Interaction with Distributed Propellers," AIAA 2014-2852, AIAA Aviation 2014.

18. M. D. Patterson, B. J. German. "Simplified Aerodynamics Models to Predict the Effects of Upstream Propellers on Wing Lift," AIAA 2015-1673, AIAA SciTech 2015.

19. M. D. Patterson, N. K. Borer, B. J. German, "A Simple Method for High-Lift Propeller Conceptual Design,” AIAA 20160770, AIAA SciTech 2016.

20. "MATLAB ${ }^{\circledR}$ - The Language of Technical Computing," http://www.mathworks.com/products/matlab/, accessed 6 May 2014.

21. J. Neter, M. H. Kutner, C. J. Nactsheim, W. Wasserman, Applied Linear Statistical Models, Fourth Edition, McGraw-Hill, Boston, MA 1996.

22. "Latin hypercube sample - MATLAB ${ }^{\circledR}$ lhsdesign," http://www.mathworks.com/help/stats/lhsdesign.html, accessed 6 May 2014.

23. R. H. Myers, D. C. Montgomery, Response Surface Methodology: Process and Product Optimization Using Designed Experiments, John Wiley and Sons, Inc., New York, NY, 1995.

24. M. Drela, H. Youngren, “AVL," http://web.mit.edu/drela/Public/web/avl/, accessed 10 May 2016.

25. M. Drela, H. Youngren, "XFOIL Subsonic Airfoil Development System," http://web.mit.edu/drela/Public/web/xfoil/, accessed 20 November 2014.

26. M. D. Patterson, B. J. German, M. D. Moore, "Performance Analysis and Design of On-Demand Electric Aircraft Concepts," AIAA-2012-5474, $12^{\text {th }}$ AIAA Aviation Technology, Integration, and Operations Conference, Indianapolis, IN, 17-19 September 2012.

27. D. Kinney, R. McDonald, "VSPAero/OpenVSP Integration," http://www.openvsp.org/wiki/lib/exe/fetch.php? media=workshop15:vspaero_2015.pdf, accessed 10 May 2016.

28. M. Drela, H. Youngren, "XROTOR Download Page," http://web.mit.edu/drela/Public/web/xrotor/, accessed 6 May 2014.

29. D. P. Raymer, Aircraft Design: A Conceptual Approach, Third Edition, American Institute of Aeronautics and Astronautics, Reston, VA, 1999.

30. J. W. Paulson, "Wind Tunnel Investigation of a Fowler Flap and Spoiler for an Advanced General Aviation Wing, NASA Technical Note TN D-8236, June 1976.

31. M. Drela, "MSES Overview," http://web.mit.edu/drela/Public/web/mses/, accessed 10 May 2016.

32. R. A. McDonald. "Modeling of Electric Motor Driven Variable Pitch Propellers for Conceptual Aircraft Design," AIAA2016-1025, AIAA SciTech, San Diego, CA, 2016.

33. "rstool - Interactive response surface modeling," http://www.mathworks.com/help/stats/rstool.html, accessed 10 May 2016.

34. M. Zeleny, Multiple Criteria Decision Making, McGraw-Hill, New York, NY, 1982.

35. A. M. Stoll, J. Bevirt, M. D. Moore, W. J. Fredericks, N. K. Borer, "Drag Reduction through Distributed Electric Propulsion," AIAA- 2014-2851, AIAA Aviation 2014, Atlanta, GA, June 2014.

36. M. D. Patterson, Conceptual Design of High-Lift Propeller Systems for Small Electric Aircraft, Ph.D. Dissertation, Georgia Institute of Technology, Atlanta, GA, August 2016.

37. Code of Federal Regulations, Title 14, "Aeronautics and Space," Part 21, "Certification Procedures for Products and Articles." Available at www.ecfr.gov, accessed 10 May 2016.

38. “OpenVSP," http://www.openvsp.org/, accessed 11 May 2016.

39. A. Dubois, M. van der Geest, J. Bevirt, "Design of an Electric Propulsion System for SCEPTOR's Outboard Nacelle," AIAA Aviation 2016, Washington, D.C., June 2016.

40. “MTV-7 Variable Pitch Propller," http://www.mt-propeller.com/pdf/datsheet/mtv-7.pdf, accessed 11 May 2016.

41. "P2006T - Aircraft Flight Manual," Doc. No. 2006/044, $3^{\text {rd }}$ Edition, Rev. 4, Construzioni Aeronautiche Tecnam S.r.l., 5 May 2014. 